\title{
Surveying the Lettuce Genome: Genome-wide Identification of Hsfs and Hsps in Response to UV and High Light Stress
}

\section{Taehoon Kim}

University of Florida

\section{Shafina Samraj}

University of Florida

Juan Jimenez

University of Florida

Celina Gómez

University of Florida

Tie Liu

University of Florida

Kevin Begcy ( $\nabla$ kbegcy.padilla@ufl.edu )

University of Florida

\section{Research Article}

Keywords: Lettuce, heat shock factors, heat shock proteins, gene duplication, light conditions

Posted Date: December 18th, 2020

DOI: https://doi.org/10.21203/rs.3.rs-126898/v1

License: () (1) This work is licensed under a Creative Commons Attribution 4.0 International License.

Read Full License

Version of Record: A version of this preprint was published at BMC Plant Biology on April 17th, 2021. See the published version at https://doi.org/10.1186/s12870-021-02959-x. 
1 Working title: Identification of Hsfs and Hsps in lettuce

3 Title: Surveying the lettuce genome: Genome-wide identification of Hsfs and Hsps in response

4 to UV and high light stress.

5

6 Taehoon $\mathrm{Kim}^{1}$, Shafina Samraj ${ }^{1}$, Juan Jimenez ${ }^{1}$, Celina Gómez ${ }^{1}$, Tie Liu ${ }^{2}, \mathrm{Kevin} \mathrm{Begcy}^{1 *}$

$8{ }^{1}$ University of Florida, Environmental Horticulture Department, Gainesville, Florida 32611

$9 \quad$ 2University of Florida, Horticultural Science Department, Gainesville, Florida 32611

10

11 ORCID IDs:

12 Taehoon Kim (0000-0002-0555-5852)

13 Tie Liu (0000-0003-0159-8270)

14 Kevin Begcy (0000-0002-5046-8029)

15

$16{ }^{*}$ Corresponding author

17 Email: kbegcy.padilla@ufl.edu

18

19 Keywords: Lettuce, heat shock factors, heat shock proteins, gene duplication, light conditions. 


\section{Abstract}

\section{Background}

25 Heat shock factors (Hsfs) and Heat shock proteins (Hsps) belong to an essential group of

26 molecular regulators involved in controlling cellular processes under normal and stress

27 conditions. The role of Hsfs and Hsps is well known in model plant species under diverse stress

28 conditions. However, in lettuce (Lactuca sativa), a highly consumed vegetable crop grown in the

29 field and in hydroponic systems, the role of these gene families in response to artificial light is

30 not well characterized.

\section{Results}

32 Using a genome-wide analysis approach, we identified $32 \mathrm{Hsfs}$ and 22 small heat shock proteins

33 (LsHsps) in lettuce, some of which do not have orthologous in Arabidopsis, poplar, and rice.

34 LsHsp60s, LsHsp90s, and LsHsp100s are highly conserved among dicot and monocot species.

35 Surprisingly, LsHsp70s have three times more members than Arabidopsis and two times more

36 than rice. Genome triplication might have contributed to the increased number of LsHsp70s

37 genes. Chromosomal distribution analysis shows larger tandem repeats of LsHsp70s genes in

38 Chr1, Chr7, Chr8, and Chr9. At the transcriptional level, some genes of the LsHsfs, LsHsps,

39 LsHsp60s, and LsHsp70s families were highly responsive to UV and high light stress, in contrast

40 of LsHsp90s and LsHsp100s which did not respond to a light stimulus.

\section{Conclusions}

42 Our genome-wide analysis provides a detail identification of Hsfs and Hsps in lettuce.

43 Chromosomal location and syntenic region analysis together with our transcriptional analysis

44 under different light conditions provide candidate genes for breeding programs aiming to 
45 produce lettuce varieties able to grow healthy under hydroponic systems that use artificial 46 light.

48 Background

49 Increasing human population, climate change conditions, decrease in water availability, and 50 pathogens and insect pressure have influenced the way we grow crops. Hydroponics is a viable 51 alternative of production to address many of these issues [1]. Vegetables and fruits are the 52 most commonly grown hydroponic crops. Within these group, lettuce (Lactuca sativa) is one of 53 the most common vegetables produced in hydroponic systems [1-3]. Some of the production 54 and health benefits of lettuce include a short production cycle, small size as well as its rich fiber, 55 vitamins, minerals, and phytochemicals content [4].

57 Lettuce is an important recently sequenced vegetable crop with a diploid genome $(2 n=2 x=18$

58 chromosomes) and an estimated size of $2.5 \mathrm{~Gb}$ [5]. A large fraction of lettuce production is 59 grown hydroponically and indoors, relying on the supply of a nutrient solution and artificial 60 light. The intensity and properties of light are critical factors that regulate photosynthesis and 61 plant growth. Optimization of nutrients and light throughout the production cycle can provide 62 better growing conditions to indoor hydroponic systems [2, 3]. However, the constant supply of 63 high-energy radiation in the form of UV or high light intensity can negatively alter not only plant 64 growth and development [6, 7], but also the transcriptional pattern of gene expression [8, 9]. 65 Under these conditions, the expression of stress responsive genes is critical to ensure plant 66 fitness and normal development. Two gene families are known to be in the front line of stress 
67 responses to tackle adverse conditions, heat shock factors (Hsfs) and heat shock proteins

68 (Hsps). Hsfs and Hsps are involved in stress response mechanisms that allow plants to control

69 folding, accumulation, and degradation of proteins.

70

71 Heat shock factors are transcriptional activators that regulate gene expression of their target

72 genes. In plants, Hsfs are classified into three groups, Hsfs type A, B, and C. Type A is the largest

73 group of Hsfs in most species including Arabidopsis (Arabidopsis thaliana), rice (Oryza sativa)

74 and poplar (Populus trichocarpa) [10-12]. Type B contains five genes and type $C$ is the less

75 diverse and contain only a few gene members. In contrast, Hsps are more abundant and are

76 classified based on their molecular weight into five subfamilies, small Hsps (sHsps), Hsp60s,

77 Hsp70s, Hsp90s, and Hsp100s. Members of Hsfs and Hsps have been described in response to

78 heat, cold, drought, and salinity in many monocots and dicots species [10-14].

80 Most studies investigating lettuce in response to light have been focused on the effects of high-

81 energy radiation with ultraviolet (UV) and high light intensity wavebands on crop quality [2] and

82 yield [3]. However, the molecular responses of Hsfs and Hsps to different light treatments have

83 not been characterized. Here, we describe a genome-wide analysis of Hsfs and Hsps genes

84 under diverse light conditions. A comprehensive description of subclasses of Hsfs, sHsps,

85 Hsp60s, Hsp70s and Hsp100s has been generated providing novel candidate genes for lettuce

86 breeding programs.

87

88 
Results

Identification and properties of Lettuce Heat Shock Transcription Factors

91 Heat shock transcription factors (Hsfs) are important transcriptional activators of stress-

92 regulated genes. Based on the recent sequencing of the lettuce genome [5], we identified

93 LsHsfs family members using an automated local BLASTP search with the previously described

94 Arabidopsis [10], rice [11], and poplar Hsfs [12] as query sequences followed by manual

95 curation. Previous Hsfs genome-wide studies have identified 21 AtHsfs, 28 OsHsfs, and 25

96 PtHsfs. Our automated search yielded 32 members of the LsHsfs family (Fig. 1A). Considering

97 that the sequenced lettuce genome is a diploid plant with an estimated genome size of $2.5 \mathrm{~Gb}$,

98 it is interesting to note that lettuce carries a higher number of Hsfs compared to rice and 99 poplar.

100

101 To further characterize LsHsfs, we collected their physiochemical features including 102 chromosomal coordinates, molecular weight (MW), theoretical isoelectric point (pl), instability 103 index, aliphatic index, hydropathicity, and predicted subcellular localization (Additional file 1: 104 Table S1). Members of the LsHsfs family showed largely variable MWs, ranging from 105 approximately $13 \mathrm{kDa}$ to $54 \mathrm{kDa}$ (Fig. 1B). Consistent with their putative function as 106 transcription factors, most of the LsHsfs were predicted to be localized in the nucleus; however, 107 LsHsfA1b and LsHsfA8 showed chloroplast signal peptides (Fig. 1B; Additional file 1: Table S1). It 108 will be interesting to investigate whether both genes could play a dual function in the nucleus 109 and the chloroplast. Another well conserved genome feature was the number of exons; LsHsfs 110 family members have between 2 to 5 exons (Fig. 1C). 
112 Because our analyses showed an increase in LsHsfs number, we decided to quantify the ratio of

113 substitution rates at non-synonymous and synonymous sites in both LsHsfs and LsHsp to

114 explore the evolutionary pressures on proteins during lettuce whole-genome triplication [5].

115 The largest dN/dS ratio was observed in LsHsfs (0.1); however, the hallmark signature of 116 positive selection is accepted to be $d N / d S>1$ [15]. All the LsHsps families showed dN/dS ratio of

1170.5 or below (Fig. 1D). In general, no evidence of positive selection was found on any other

118 member of the LsHsfs and the different LsHsps families based on the dN/dS ratio analysis (Fig.

119 1D; Additional file 2: Table S2).

121 Classification, gene structure, and phylogenetic analysis of lettuce Hsfs

122 Since Arabidopsis Hsfs have been well characterized, we used their nomenclature to classify

123 lettuce Hsfs. As the criteria, we selected sequences with an E-value threshold of $1 \mathrm{E}^{-20}$. LsHsfs

124 were classified in three main groups (Fig. 2A); type A (13 genes), type B (12 genes) and type C (7

125 genes). To further assess the evolutionary relationship of the Hsfs, a phylogenetic tree was

126 constructed based on the full-length amino acid sequences from both lettuce and Arabidopsis

127 (Fig. 2B). While most members of the type A subfamily in Arabidopsis had a similar number of

128 orthologous lettuce genes, we found only two members (LsHsfA1a and LsHsfA1b) orthologous

129 with four Arabidopsis Hsf genes in the subgroup A1. LsHsfs Type B and C subfamilies showed a

130 larger number of new members compared to Arabidopsis. Interestingly, we identified a new

131 lettuce subgroup that is not present in Arabidopsis, that we named LsHsfB5. It has three 132 members with high homology among them. Remarkably, we also identified a large number of 
133 LsHsf Type C (seven genes) compared to Arabidopsis (one gene), which mainly contributed to

134 the overall increase in number of the LsHsfs family (Fig. 2B). The gene structure of the LsHsfs

135 was highly conserved; however, four members of the LsHsfs type C (LsHsfC1a, LsHsfC1b,

136 LsHsfC1d, LsHsfC1e) and three of the LsHsfs type B (LsHsfB1a, LsHsfB1b, LsHsfB1c) family

137 displayed different gene structures and variable motif distributions (Fig. 2C).

139 Lettuce Small Heat Shock Proteins (LssHsps)

140 We identified and classified lettuce small heat shock proteins (LssHsps) into nine subfamilies

141 (Fig. 3A). Each subfamily was named based on their predicted MW and subcellular localization

142 (Fig. 1B). Physiochemical features and predicted subcellular localization were also characterized

143 (Additional file 1: Table S1). Five lettuce sHsp subfamilies are cytosol-localized (C-I, C-II, C-III, C-

144 IV, and C-V). The other four subfamilies are predicted to be expressed in the endoplasmic

145 reticulum (ER), peroxisome (PX), chloroplast (CP), and mitochondria (MT) (Fig. 3A-B). We found

146 a large number of members belonging to the C-I (12 genes) and C-II subfamilies ( 6 genes) in the

147 lettuce genome compared to only six C-I and two C-II genes in the Arabidopsis genome (Fig. 3B).

148 Interestingly, we did not find LsHsp-CVI members based on their homology with Arabidopsis

149 (Fig. 3B). Remarkably, the large majority of LssHsps have a single exon (Fig. 3C); nonetheless,

150 the members of the LssHsp-PX, LssHsp-P, and LssHsp-M subclades have two or three exons. At

151 the gene structure level, the exon/intron structures of LssHsps were relatively uniform except

152 for LsHsp23.8-Cl and LsHsp37.6-ER which contain long introns spanning $7.77 \mathrm{~kb}$ and $9.16 \mathrm{~kb}$,

153 respectively (Fig. 2C). In addition, each subfamily of LssHsps has its unique number of

154 conserved motifs. Remarkably, LssHsps subfamilies predicted to be expressed in the cytosol 
155 showed distinctive motifs (Motif 4) which could be an indication of their potential function in 156 the plant cell (Fig. 3C).

158 LsHsp60, a well conserved gene family

159 Based on the phylogenetic relationships of the lettuce Hsp60 family with Arabidopsis Hsp60s, 160 we classified LsHsp60 family into four subclasses: Cpn60 which contains 12 cytoplasmic genes,

161 Cpn60 $\alpha$ having four chloroplastic members, Сpn60 $\beta$ containing three chloroplastic members, 162 and the Hsp60 subclass consisting of three mitochondrial genes (Fig. 4A). In general, Hsp60

163 genes are well conserved between Arabidopsis and lettuce given that each Arabidopsis Hsp60

164 member has at least one orthologous gene in lettuce. In addition, there are no Hsp60 lettuce-

165 specific clade (Fig. 4B). At the gene structure level, the LsHsp60 has a well conserved motif 166 structure; however, the number of exons varies across the different subfamilies (Fig. 4C). A 167 physiochemical characterization of LsHsp60 was also conducted and can be found in Additional 168 file 1: Table S1.

170 Another interesting feature of the LsHsp60 family is the variability in their intron phase. Based

171 on the disruption of the last codon of an exon, introns have been divided into three types. The

172 first is called phase 0 , in which introns do not disrupt a codon. The second is called phase 1 , in 173 which introns disrupt a codon between the first and second bases. Finally, phase 2, where 174 introns disrupt a codon between the second and third bases [16]. Unlike LsHsfs and the other 175 LsHsps families, every LsHsp60 gene has at least one phase 0 and phase 2 intron, with the only 176 exception of LsCpn60-8 which does not have any introns (Fig. 4C). 


\section{Tandem gene duplication of Lettuce Hsp70s}

179 Hsp70s are molecular regulators of stress responses, as they maintain protein homeostasis by

180 mediating folding or protein denaturation [17]. Hsp70 family is comprised of DnaK subfamily

181 including cytoplasmic Hsp70 (Hsp70), truncated Hsp70 (Hsp70t), plastidic Hsp70 (cpHsp70),

182 mitochondrial Hsp70 (mtHsp70), endoplasmic reticulum-localized immunoglobin binding

183 protein (BiP), and the Hsp110/SSE subfamily [18]. We identified nine Hsp110/SSE genes, one

184 Hsp70t gene, three cpHsc genes, two mtHsc genes, and two BiP genes in lettuce (Fig. 5A).

185 Strikingly, the lettuce genome contained 47 cytosolic Hsp70 genes (Fig. 5A-B), which greatly

186 increased the number of the Hsp70 family genes (64 genes) compared with 18 genes found in

187 Arabidopsis (Fig. 1A). From the 47 members of the cytosolic Hsp70, only three of them have

188 orthologous in Arabidopsis, the remaining 44 members are novel lettuce Hsp60s (Fig. 5A-B).

189 Another LsHsp70 subclass in which we identified new members was the LsHsp110/SSE (Fig. 5B).

190 In Arabidopsis, four members of the Hsp110/SSE have been described. Our analysis yielded nine

191 members, in which five are clustered together and do not have orthologues in Arabidopsis (Fig.

192 5B).

193 An interesting gene feature of the Hsp70 subfamily is the low number of exons and the well

194 conserved motif organization. The LsHsp70t subclass single member has no introns and a few

195 motifs, differently than all the other subclasses. Cytosolic Hsp70 genes have one intron and a

196 well conserved motif distribution (Fig. 6A). However, some of the residues are not well

197 conserved, except for motifs 5, 7, 8, 15, 16, 19, and 20 (Fig. 6B). Taken together, our results 
198 show a large increase in gene number in the LsHsp70 family, which could be related to stress

199 responsiveness to environmental signals.

201 Heat Shock Protein 90 and 100

202 Hsp90s and Hsp100s are the two heat shock protein subfamilies with the highest molecular

203 weight (Fig. 1B). Unlike the other LsHsps families, both LSHsp90 and LsHSp100/ClpB families did

204 not increase in gene number during the lettuce whole-genome triplication in comparison with

205 Arabidopsis, rice, and poplar (Fig. 1A). Remarkably, 62\% of the LsHsp100s were localized in

206 chloroplasts, $26 \%$ in cytoplasm, and $12 \%$ were mitochondrial localized (Fig. 1B). LsHsp90s

207 showed a more diverse localization in several subcellular compartments including ER, nucleus,

208 cytoplasm and chloroplast as well as physiochemical features including $\mathrm{MW}$, pl, and

209 hydropathicity (Fig. 1B; Additional file 1: Table S1).

210

211 Plant Hsp90 family proteins have been classified into two main groups (group I and II), and are

212 divided into four subclasses (group la, Ib, Ila, and IIb) [19]. Similarly, LsHsp90s subfamily contain

213 seven members, organized in four subclasses. Group la includes LsHsp90-1a and LsHsp90-1b

214 (Fig. 7A), both orthologous genes of a single member described in Arabidopsis. Similarly, group

215 Ib contains only two members orthologous of AtHsp90-2/3/4 group (Fig. 7B). At the gene

216 structure level, LsHsp90s can be divided in two main groups based on their exon and motif

217 numbers (Fig. 7C). All the members of the group la and Ib have three exons and a highly similar

218 motif structure. In contrast, members of the group Ila and IIb have a larger number of exons (19 
219 and 12 exons, respectively) and a different motif patten compared to the group la and $\mathrm{Ib}$, but

220 similar among them (Fig. 7C).

221 LsHSp100/ClpB family contains five subclasses (Fig. 7D), CplB-Cyt (cytoplastic localized), CplB-CP

222 (chloroplast localized), ClpB-MT (mitochondrial localized), ClpC, and ClpD. Phylogenetic analysis

223 shows that lettuce and Arabidopsis shared a comparable number of group members with

224 similar localization (Fig. 7E). Lettuce Hsp100 family genes also showed structural similarities

225 depending on their subclasses. Cytoplasmic ClpB genes included only two exons; however,

226 chloroplastic and mitochondrial $\mathrm{ClpB}, \mathrm{ClpC}$, and $\mathrm{ClpD}$ genes contained between eight to twelve

227 exons (Fig. 7F).

228

229 Chromosomal distribution and duplication events of Hsf and Hsp genes in lettuce

230 In our initial analysis, we found an increased number of Hsfs and Hsps in lettuce compared to

231 other species (Fig. 1A). To further investigate which particular Hsfs and Hsps subfamilies

232 underwent duplication or genome lost, we first analyzed their chromosomal distribution. A

233 total combined of $165 \mathrm{Hsfs}$ and Hsps were randomly distributed in each of the nine lettuce

234 chromosomes (Fig. 8). A syntenic analysis of lettuce against other plant species of the Asterid

235 order suggested a whole-genome triplication event in lettuce since its divergence from the

236 grape lineage [5]. Using syntenic regions previously categorized [5], we searched for Hsps and

237 Hsfs located in those regions (Fig. 8). Remarkably, 43.7\% (14 out of 32) of the LsHsfs were

238 located in syntenic regions, suggesting their importance during the whole-genome triplication

239 process. Syntenic region A was found in chr1, chr2, and chr6 and only contained Hsps subfamily

24060 in particular of the subclass LsCpn60. Syntenic region B is present in chr1, chr4 and chr5 and 
241 carries mostly members of the LsClp (LsHsp100). Syntenic region D carries LsHsfB1a in chr3 and

242 LsHsfB1b in chr4. It was also present in chr2; however, no other LsHsf was located in that

243 syntenic region (D). Syntenic region E was more heterogenous and it is present in chr2, chr8,

244 and chr9. It carries LsHsfs and members of the LsHP70 and LsHsp90. Interestingly, syntenic

245 region $G$ was only located in chr3 carrying LsHsfA1A and LsHsfB2b, while chr6 carries LsBiP1 and

246 LsBiP2 (LsHsp70). Syntenic region I contain LscpHsc70-1 and LsmtHsc70-1 in chr5 and their

247 duplicated genes LscpHsc70-3 and LsmtHsc70-2 were found in chr9 respectively. Syntenic

248 region $\mathrm{H}$ carries a diverse mix of LsHsfs and LsHsps and it is located in chr5, chr8 and chr9.

249 Finally, syntenic regions C, F and $\mathrm{H}$ do not carry any Hsfs and Hsps.

250 To further elucidate whether LsHsps were localized as gene clusters within the lettuce genome,

251 we searched for regions containing two or more Hsps genes within a $200 \mathrm{~kb}$ region (Additional

252 file 4: Table S4). The large majority of gene clusters found in the lettuce genome represent

253 genes within the LsHsp70s subfamily (Fig. 8), and are distributed in chr1, chr4, chr5, chr7, chr8,

254 and chr9. Interestingly, chr1 and chr9 carry gene clusters with nine and ten LsHsps70s genes,

255 respectively. This indicates the greater number of Hsp70 genes found in lettuce compared to

256 Arabidopsis, rice, and poplar could be the result of clustered tandem duplication. Similarly, chr8

257 carries two gene clusters of LsHsp70s. A second group of abundant gene clusters found in the

258 lettuce genome belong to the LssHsps family. Tandem repeat of LssHsps gene clusters were 259 found in chr2, chr7, chr8, and chr9. Our results showed that LsHsfs and LsHsps70s were 260 fundamental genes during the whole-genome triplication in lettuce. 
263 In order to understand how LsHsfs and LsHsps are regulated, we performed a Cis-regulatory

264 element analysis of LsHsfs and LsHsps promoters using a $2 \mathrm{~kb}$ upstream region of each gene.

265 We particularly focused on Cis-regulatory elements related to light, stress, and hormone

266 responses as well as metabolism, cell cycle, and circadian rhythm (Fig. 9; Additional File 5: Table

267 S5). A total number of 59 cis-regulatory elements were identified among LsHsfs and LsHsp

268 genes. Interestingly, within the light responsive cis-elements, G-box, GT1 motif, TCT motif, and

269 box 4 were highly abundant in all LsHsf and LsHsp gene families. ARE cis-element was the most

270 abundant in the stress responsive category, having multiple ARE motif present across the

271 promoter of both LsHsfs and LsHsps genes (Fig. 9; Additional File 5: Table S5). In the hormone

272 stress category ABRE was ubiquitously present in the LsHsfs promoters and in most of the LsHsp

273 promoters. However, ABRE motif was underrepresented in promoter regions of LsHsp90s (Fig.

274 9; Additional File 5: Table S5). Cis-elements related to metabolism, cell cycle and circadian

275 rhythm were less abundant and indicate the low involvement of LsHsfs and LsHsps in these

276 biological processes.

278 Transcriptional analysis of LsHsfs and LsHsps under different light conditions

279 Many studies have shown that Hsfs and Hsps genes are involved in plant responses to abiotic 280 stresses in model plants [10] and other crop species [11, 14, 20, 21]. To investigate the role of

281 LsHsfs and LsHsps under different light conditions, we grew lettuce plants $c v$. Codex in

282 hydroponic conditions. After germination, lettuce plants were grown for 10 days under broad-

283 spectrum LED lamps providing an average of $150 \pm 5 \mu \mathrm{mol} \cdot \mathrm{m}^{-2} \cdot \mathrm{s}^{-1}$ light unit. Then, light 284 conditions were adjusted to $220 \pm 5 \mu \mathrm{mol} \cdot \mathrm{m}^{-2} \cdot \mathrm{s}^{-1}$ light for 22 days. Control plants were kept at 
285 the same light conditions while UV treated plants were exposed to $220 \mu \mathrm{mol} \cdot \mathrm{m}^{-2} \cdot \mathrm{s}^{-1}$ 286 supplemented with UV radiation. High light condition was achieved by providing $287440 \pm 5 \mu \mathrm{mol} \cdot \mathrm{m}^{-2} \cdot \mathrm{s}^{-1}$. All end-of-production treatments were applied for 4 days and then 288 mature lettuce plants were harvested for gene expression analysis (Fig. 10A). In general, lettuce 289 plants under UV and high light conditions became reddish while lettuce leaves retained a green 290 color under control conditions (Fig. 10B).

292 We selected representative members of each subfamily of LsHsfs and LsHsps based on our 293 genome-wide analysis. In general, LsHsfs types A, B, and C were highly responsive to UV and 294 high light stress. LsHsfA1 and LsHsfA7c, type A Hsfs, showed significant downregulation when 295 lettuce plants were exposed to UV radiation and high light conditions (Fig. 10C). In contrast, 296 LsHsfB1a was only downregulated in response to high light but did not respond to UV radiation. 297 In the case of Hsfs type C, we analyzed the expression of LsHsfC1c and observed an 298 upregulation in response only to UV radiation (Fig. 10C).

299 Almost all subclasses of LsHsps were upregulated in response to at least one of the treatments, 300 UV radiation or high light. Interestingly, members of the LsHsp-CP and LsHsp-PX subclasses 301 showed the highest upregulation in response to UV treatment (Fig. 10C). Transcriptional 302 expression of LsHsp60s under UV radiation and high light showed a wide range of responses. 303 Although LsCpn60-2, LsCpn60a4 LsCpn60-1 did not respond to neither of the treatments, 304 LsCpn60-4a was upregulated and LsCpn60a3 downregulated for both treatments (UV radiation 305 and high light) (Fig. 10C). 
306 In relation of LsHsp70s, all the novel members identified were responsive to both treatments

307 (UV and high light stress); however, high light was the most effective at downregulating their

308 expression (Fig. 10C). Interestingly, members of the cpHsc and Hsp110/SSE subclasses did not

309 show any transcriptional response. Remarkably, LsHsp90 and LsHsp100 did not respond to UV

310 and high light treatment, suggesting that they might not be involved in response to light

311 conditions.

312

313 Since we found tandem duplication of several LsHsps genes, we selected several of those genes

314 to explore the possibility of cluster expression in response to light conditions. Surprisingly, we

315 did not find any transcriptional response of duplicated genes based on our RT-qPCR data (Fig.

316 10C). Interestingly, LsHsp18.2-CII did not respond to light stress; however, LsHsp18.4b-CII

317 located in the same duplicated cluster was significantly upregulated in response to high light.

318 Another example was the case of $\mathrm{LsCpn60 \alpha 3}$ and $\mathrm{LsCpn60 \alpha 4}$ in which the former was

319 significantly downregulated in response to both treatments, but the latter did not change

320 transcriptionally (Fig. 10C). Because most of the new LsHsp70s members that we identified are

321 located in clusters (Fig. 8), we tested three of these clusters containing LsHsp70s genes (Fig.

322 10C). We found that none of the genes tested show similar transcriptional pattern. It is worth

323 noticing that functional characterization of those clusters might provide a better understanding

324 of their regulatory role.

325

326 Discussion 
327 Plant stress responses are frequently the result of simultaneous mechanisms that synergistically

328 and/or coordinately operate to prevent, maintain, and re-establish cellular homeostasis [22-

329 24]. One of the best characterized stress mechanisms is the heat shock proteins response. It has

330 been demonstrated that Hsps play an important role in many cellular processes in response to

331 stressful conditions, which may disclose whole plant responses to multiple environmental

332 stresses. This particular stress response mechanism involves the activation of different Hsp

333 families [17, 21] regulated by Hsfs to produce a rapid and constitutive response. Thus, the

334 different classes of Hsps work together with Hsf to maintain cellular homeostasis $[10,17]$. In

335 this study, we performed a genome-wide analysis for Hsfs and Hsps in lettuce and identified an

336 overall increase in most of these gene families.

\section{Diversification of LsHsfs}

339 The composition of the Hsfs family has so far been fully described in model plant species as well

340 as in some cereal crops [10-12, 20, 25]. There are $21 \mathrm{Hsfs}$ in Arabidopsis (Arabidopsis thaliana),

$34126 \mathrm{Hsfs}$ in tomato (Solanum lycopersicum), 25 in both rice (Oryza sativa) and maize (Zea mais)

342 and 52 in soybean (Glycine $\max$ ) [13, 25-27]. However, several studies have shown that Hsfs is

343 subject to evolutionary changes based on both number and composition of the Hsf family [27].

344 Our genome-wide analysis in lettuce identified $32 \mathrm{Hsfs}$ genes; a higher number compared to

345 Arabidopsis (Fig. 2A-B). This increase in gene number might have resulted in evolutionary

346 advantage during the whole genome triplication [5]. Interestingly, unlike Hsps, almost all Hsfs

347 are not located in tandem repeats in the lettuce genome, with the exception of LsHsf5b and 
348 LsHsf5c which are positioned in tandem repeat in the chr7 (Fig. 8), suggesting that all LsHsfs are

349 functional genes with a possible role in the stress response and homeostasis maintenance.

350 The basic structure of Hsf proteins is formed by a DNA binding domain, oligomerization domain,

351 and a C-terminal activation domain. Classification of plant Hsfs (A, B, and C) is mainly based on

352 variances in these three domains, particularly the oligomerization domain. The most obvious

353 differences are the insertion of additional amino acid residues in the oligomerization domain of

354 type $\mathrm{A}$ and type $\mathrm{C} \mathrm{Hsfs}$ and the absence of transcriptional activator motifs in the C-terminal

355 domain of type B and C Hsfs [20, 25]. In Lettuce, all the Hsfs follow the same basic structure. For

356 instance, the type $\mathrm{C}$ members lack C-terminal domains resulting in smaller genes (Fig. 2C). Type

357 A Hsfs are known to function as transcriptional activators of stress genes. Genetic and

358 functional analysis suggests that HsfA1a and HsfA1b are central regulators required in the early

359 phase of the heat shock response $[22,24,28]$. In Arabidopsis, members of the Hsfs type A

360 function as the main positive regulators in heat shock-responsive gene expression including

361 members of the Hsp70 and Hsp90 families [29, 30]. We observed a strong downregulation of

362 LsHsfA1a under UV and high light (Fig. 10), which might explain the lack of response of

363 LsHsp90s and LsHsps100s to light stress conditions.

\section{Duplications play major role in the diversification of the LsHsp70 family}

366 In plants, gene duplication through whole-genome duplication (WGD), tandem or segmental

367 events represent a major force driving gene family expansion [31, 32]. Similarly as described in 368 many other plant species $[10,11]$, lettuce Hsfs and Hsps have experienced complex biological 369 rearrangements, resulting in adaptation to specific conditions (Fig. 8) [5]. Our phylogenetic 
370 analysis comparing LsHsp70s with Arabidopsis, rice, and poplar revealed six different groups,

371 designated as cytosolic Hsp70, Hsp70t, BiP, MtHsc, cpHsc and Hsp110/SSE (Fig. 5B). We found

372 that the LsHsp70 family was the one that had undergone the largest duplication within the

373 Hsps. Out of 64 LsHsp70 gene members 55\% (36 members) are localized in duplicated blocks in

374 the lettuce genome. Our findings suggest that prior to the whole genome triplication, LsHPS70

375 suffered dynamic rearrangements that allowed the emergence of novel members of the

376 LsHsp70 family (Fig. 5). Thus, the ancient genetic rearrangement in the LsHsp70 family might

377 have resulted from the whole-genome triplication as an essential genetic group of this plant

378 species. Interestingly, $39 \%$ of the LsHsp70s have a single exon, all of them from the highly

379 abundant cytosolic Hsp70 subclass (Fig. 6), which is in line with previous studies which suggest

380 that the evolution of several plant gene families is associated with the diversification of exon

381 and intron within those gene family members [33].

\section{Light quality effects on LsHsfs and LsHsps transcriptional levels}

384 During a plant life's cycle, a large number of essential processes are regulated by light. These

385 processes include photosynthesis, plant growth and development, anthocyanin biosynthesis,

386 and gene expression, among others [2, 3, 34, 35]. In Arabidopsis, three A-type Hsfs, HsfA1D,

387 HsfA2, and HsfA3 were found to regulate early responses during excess light energy [36]. In

388 lettuce, under UV and high light, we found strong downregulation of LsHsfA1a and LsHsfA7c

389 (Fig. 10C). In addition, LsHsfB1a was downregulated in response to high light and LsHsfC1c

390 upregulated in response to UV. Interestingly, under different light conditions, most studied

391 members of the Lettuce Hsp90 and Hsp100 families were not regulated, while members of the 
392 LssHsp and LsHps60 families were significantly up-regulated (Fig. 10C). While further studies

393 need to be conducted to elucidate the functions of these genes under UV and high light

394 conditions, our data suggests that the hydroponic conditions we used in this study are not able

395 to trigger LsHsp90s and LsHsp100s molecular responses.

396

397 The specific response to UV and high light of many members of the LsHsp70 family might be 398 associated with the increased member duplication of the cytosolic Hsp70 in lettuce. Under 399 excessive light energy, plants first try to dissipate excess light stored as electron energy in the 400 chloroplasts to avoid heat damage [37]. Under UV conditions, we observed a high 401 transcriptional expression of Hsfs and Hsps. For instance, a strong downregulation of LsHsp7040228 and LsHsp70t-1 specially to high light conditions but a significant upregulation of these 403 under UV (Fig. 10C). Our study provides initial assessment of Hsfs and Hsps under different light 404 conditions, However, the precise regulatory mechanisms under hydroponic conditions under 405 control and stress conditions require further investigation.

\section{Conclusions}

408 Our detailed genome-wide analysis on the heat shock factors and heat shock proteins in lettuce 409 identified 32 and 165 genes, respectively. Phylogenic analysis Hsfs and Hsps genes highlight a 410 close relationship with their orthologous genes in Arabidopsis. Interestingly, a large number of 411 novel members were also found in Hsfs, sHsps and Hsp70 gene families probably as result of 412 the genome wide triplication. Several Hsfs, sHsps, Hsp60s, and Hsp70s genes are highly 413 responsive to UV and high light conditions and provide candidates for breeding programs 
414 aiming to produce lettuce varieties able to grow healthy under hydroponic systems that use

415 artificial light.

417 Methods

418 Plant material and growth conditions

419 Lettuce seeds of red-leaf cultivar cv. Codex $r z$ were pre-germinated for 48 hours until radicle 420 emergence was observed. Germinated seeds were transplanted into rockwool plugs [2].

421 Seedlings were propagated for 10 days inside a walk-in growth chamber using an average 422 photosynthetic photon flux of $150 \pm 5 \mu \mathrm{mol} \cdot \mathrm{m}^{-2} \cdot \mathrm{s}^{-1}(24-\mathrm{h}$ photoperiod) and a daily light integral 423 (DLI) of $12.96 \mathrm{~mol} \cdot \mathrm{m}^{-2} \cdot \mathrm{d}^{-1}$ provided by broad-spectrum LED lamps (Philips GP150 Red/Deep

424 Red/Blue Low Blue High Output). Temperature and relative humidity (RH) were set at $24^{\circ} \mathrm{C}$ and $42560 \%$ to $80 \%$, respectively; $\mathrm{CO}_{2}$ concentration was maintained at ambient levels. Seedlings of 426 similar size were transplanted into individual deep-water culture closed hydroponic systems 427 using plastic cups of $5 \mathrm{~cm}$ of diameter. Plants were grown using a commercial water-soluble 428 fertilizer (OASIS ${ }^{\circledR}$ Grower Solutions Hydroponic Fertilizer 16-4-17) dissolved in tap water at a 429 concentration of $150 \mathrm{mg} \cdot \mathrm{L}^{-1} \mathrm{~N}\left(\mathrm{EC}\right.$ and $\mathrm{pH}=$ approx. $1.2 \mathrm{dS} \cdot \mathrm{m}^{-1}$ and 6.0 , respectively). Each $7.6 \mathrm{~L}$

430 hydroponic system had a clear plastic tube attached to an air pump to provide continuous 431 aeration.

\section{Light treatments}

434 Lettuce plants were grown inside a growth chamber equipped with two opposite multi-level 435 shelves. Each shelf was considered a block with four levels used as treatment compartments. 
436 All compartments held four hydroponic systems. Plants were grown under broad-spectrum LED

437 lamps providing an average DLI of $15.84 \mathrm{~mol} \cdot \mathrm{m}^{-2} \cdot \mathrm{d}^{-1}\left(220 \pm 5 \mu \mathrm{mol} \cdot \mathrm{m}^{-2} \cdot \mathrm{s}^{-1} ; 20-\mathrm{h}\right.$ photoperiod 438 from 02:00 to 22:00 h). The average ambient day (from 02:00 to 22:00 h) and night (from 22:00

439 to $02: 00 \mathrm{~h}$ ) air temperature of the chamber was set at $22^{\circ} \mathrm{C}$ and $21^{\circ} \mathrm{C}$, respectively; $\mathrm{CO}_{2}$ 440 concentration, and $\mathrm{RH}$ was set at $405 \mathrm{ppm}$ and $60 \%$ to $80 \%$, respectively. After 22 days in the 441 chamber, plants were subjected to one of three treatments during 4 days: end-of-production 442 (EOP) UV-A $\left(11 \mu \mathrm{mol} \cdot \mathrm{m}^{-2} \cdot \mathrm{s}^{-1}\right)+$ white light $\left(220 \mu \mathrm{mol} \mathrm{m} \mathrm{m}^{-2} \cdot \mathrm{s}^{-1}\right)$, EOP high light $\left(440 \pm 5 \mu \mathrm{mol} \cdot \mathrm{m}^{-2} \cdot \mathrm{s}^{-}\right.$ $\left.443{ }^{1}\right)$ and a control $\left(220 \pm 5 \mu \mathrm{mol} \cdot \mathrm{m}^{-2} \cdot \mathrm{s}^{-1}\right)$ with no EOP treatment (Fig. 10A).

445 Sequence retrieval and phylogenetic reconstruction

446 Heat Shock Factors (Hsfs) and Heat Shock Proteins (Hsps) sequences of Arabidopsis, rice and 447 poplar [10-12] were retrieved from public databases. When a gene had splice variants, primary 448 transcripts were selected as representative for the gene. To identify putative lettuce Hsfs and 449 Hsps, retrieved sequences were used as queries using an automated BLASTP search against the 450 lettuce genome database in Phytozome [38]. An E-value threshold of $1 \mathrm{E}^{-20}$ was used. When 451 multiple transcripts were predicted for a locus from BLASTP, a transcript with the lowest 452 E-value was chosen as a representative for the locus.

453 Phylogenetic trees were generated by the Maximum Likelihood method and Le_Gascuel_2008 454 model [39] with 500 Bootstrap replications using MEGA X software [40] as previously described 455 [41]. A discrete Gamma distribution was used to model evolutionary rate differences among 456 sites. Gene names of lettuce Hsfs and Hsps were assigned based on their phylogenetic 457 relationship with Arabidopsis proteins. Lettuce sHsps were classified according to their 
458 molecular weights obtained using the ProtParam tool [42]. Phylogenetic analysis data was

459 deposited in the repository of phylogenetic information, TreeBASE and it is available in the 460 following link: $\quad$ http://purl.org/phylo/treebase/phylows/study/TB2:S27434?x-access-

461 code=f2368699e8e38ce6d23cad64f44dc452\&format=html

463 Physical and chemical properties of Heat shock factors and heat shock proteins

464 The physical and chemical properties including molecular weight, isoelectric point, instability

465 index, aliphatic index, and hydropathicity of all lettuce Hsfs and Hsps were estimated using

466 ProtParam tool from ExPASy server [42]. Protein subcellular localization of lettuce Hsfs and

467 Hsps were predicted using WoLF PSORT [43]. Multiple sequence alignment for Arabidopsis and

468 lettuce Hsfs and Hsps were performed by MUSCLE [44].

470 Chromosomal mapping and characterization of tandem duplication events of Hsf and Hsp

471 genes in the lettuce genome

472 The chromosomal locations of Lettuce Hsfs and Hsps genes were retrieved from Phytozome, 473 Lactuca sativa V8 genome database [38]. Homologous chromosome segments resulting from

474 whole-genome triplication events were identified as described previously [5]. Segmental gene 475 duplication events were represented according to the phylogenetic relationship among 476 adjacent genes and sequence similarity ( $\geq 70 \%)$. Tandem duplication was evaluated based on

477 the following criteria: genes within a 200-kbp region in a chromosome with the nucleotide 478 sequence similarity $\geq 70 \%$. Sequence similarity was calculated using the EMBOSS Water with 479 Smith-Waterman local alignment algorithm [45]. 
481 Non-synonymous (dN) to synonymous substitution (dS) ratio (dN/dS) analysis

482 The Ratio between non-synonymous mutations (dN) to synonymous mutation (dS) of each

483 lettuce Hsf or Hsp gene and their corresponding Arabidopsis ortholog was calculated using 484 EMBOSS Water pairwise alignment [45]. The $\mathrm{dN} / \mathrm{dS}$ ratio was estimated by utilizing the 485 PAL2NAL tool with the input of the pairwise DNA sequence alignment [46].

487 Gene structure, conserved motif and Cis-regulatory element analysis of LsHsf and LsHsp genes

488 Exon-intron structures of lettuce Hsfs and Hsps were depicted using the Gene Structure Display

489 Server - GSDS v2.0 [47]. Genomic sequence and coding sequences of each Hsf or Hsp gene 490 were downloaded from Phytozome database and aligned to predict the exon-intron structure.

491 Conserved motifs analysis of Hsfs and each Hsp family were determined using Multiple Em for

492 Motif Elicitation (MEME) Suite online program with the following parameters: maximum motif

493 numbers = 20; site distributions = any number of repetitions; motif width $=6$ to 50 [48].

494 Cis-regulatory elements of Lettuce Hsf and Hsp genes were identified using 2,000 bp upstream 495 regions of each lettuce Hsf and Hsp genes using the PlantCARE database [49].

497 RNA isolation and RT-qPCR

498 Total RNA from leaves of twenty-two days old mature plants was isolated using Trizol (Ambion) 499 following the manufacturer's instructions. cDNA synthesis was performed using reverse 500 transcription system (Invitrogen SuperScript II) and oligo(dT) primers. Real-time PCR reactions 501 were performed using SYBR Green FastMix (Quantabio) as previously described [50]. Lettuce 
502 Tubulin (LsTUB) was used as housekeeping gene for internal normalization control. qPCRs were

503 performed in the QuantStudio ${ }^{\mathrm{TM}} 3$ Real-Time PCR System (Applied Biosystems) in a 96-well

504 reaction plate. Primers used are described in Additional File 6: Table S6. Cycling parameters

505 consisted of $5 \mathrm{~min}$ at $95^{\circ} \mathrm{C}$, and 45 cycles of $95^{\circ} \mathrm{C}$ for $15 \mathrm{~s}, 60^{\circ} \mathrm{C}$ for $30 \mathrm{~s}$, and $72^{\circ} \mathrm{C}$ for $30 \mathrm{~s}$ [51].

506 RT-qPCR reactions were performed in triplicate for each RNA sample on three biological

507 replicates of each light condition. Specificity of the amplifications was verified by a melting

508 curve analysis. Relative amounts of mRNA were calculated from threshold points (Ct values)

509 located in the log-linear range of real-time PCR amplification plots using the $2^{-\Delta \Delta C t}$ method [52].

\section{Statistical analysis}

$511 \mathrm{R}$ software/environment was used for the statistical analyses of the RT-qPCR data. Three

512 independent experiments in which each experiment had four biological replicates were used. t-

513 test was used to compare gene expression between the experimental groups (UV and high

514 light) and the control group (normal light). Differences in means were considered significant at

$515 p$-value $<0.05$

\section{Declarations}

518 Ethics approval and consent to participate

519 Not applicable

520 Consent for publication

$521 \quad$ Not applicable

522 Availability of data and materials 
523 All data generated or analyzed during this study are included in this published article and its

524 supplementary information files.

\section{Competing interests}

526 The authors declare that they have no competing interests

527 Funding

528 This work was supported by the Competitive Seed Grant Research Initiative (Grant No.

529 00129910) from the College of Agricultural and Life Sciences at the University of Florida to K.B.

530 We also acknowledge Kalera Inc. for funding the hydroponic experiments.

\section{Authors' contributions}

532 T.K. and S.S performed the bioinformatics analysis. T.K. and J.J performed the experiments. C.G.

533 provided plant material. T.L provided equipment and reagents. K.B. conceived the experiments.

534 T.K and K.B. analyzed the data. T.K. prepared the figures. KB wrote the manuscript with input

535 from T.K. All authors read and approved the manuscript.

536 Acknowledgements

537 Not applicable

538 Authors' information (optional)

539 T.K; S.S; J.J; C.G and K.B from University of Florida, Environmental Horticulture Department,

540 Gainesville, Florida. T.L from University of Florida, Horticultural Science Department,

541 Gainesville, Florida. 
547 Figure Legends

548 Fig. 1. General gene structure features of lettuce Hsfs and Hsps. (A) Numbers of Hsf and Hsp

549 genes in lettuce, Arabidopsis, poplar and rice. (B) Molecular weight analysis of LsHsfs and

550 LsHsps. (C) Exon number distribution of the lettuce Hsfs and Hsps. (D) Evaluation of positive

551 selection of LsHsfs and LsHsps families based on the dN/dS ratio analysis.

552 Fig. 2. Phylogenetic analysis, exon-intron structure, and motif distribution of lettuce heat

553 shock transcription factor (LsHsf) gene family. (A) List of lettuce Hsf genes. (B) Phylogenetic

554 relationship of Hsf proteins of lettuce and Arabidopsis. LsHsf and AtHsf members in each

555 subfamily (Type A, Type B, and Type C) are represented in different colors. (C) Gene structure

556 and motif composition analysis of $L s H s f$ genes with blue boxes, green boxes, and black lines

557 indicate untranslated region (UTR), coding sequence (CDS), and intron, respectively. The

558 numbers on each intron represent the intron phase $(0,1$, or 2$)$. Detailed information of all

559 motifs is shown in Additional file 3: Table S3.

560 Fig. 3. Identification, phylogenetic and gene structure analysis of lettuce small heat shock

561 protein (LssHsp) gene family. (A) List of sHsp genes in lettuce. Classification of LssHsp genes

562 were assigned according to their molecular weight and subcellular localization (Additional Table

563 S1). (B) Phylogenetic relationship of LsHsp proteins and A. thaliana. LssHsp proteins are marked

564 in bold. LssHsp and AtsHsp members in each subfamily (C-I, C-II, C-III, C-IV, C-V, C-VI, ER, MT, CP,

565 and PX) are represented in different colors. (C) Gene structure and motif composition analysis

566 of LssHsp genes. Blue boxes, green boxes, and black lines indicate untranslated region (UTR), 
567 coding sequence (CDS), and intron, respectively. The numbers on each intron represent the

568 intron phase $(0,1$, or 2$)$. Detailed information of all motifs is shown in Additional file 3: Table 569 S3.

570 Fig. 4. Classification, evolutionary relationship, gene structure and motif distribution of

571 lettuce heat shock protein 60 (LsHsp60) gene family. (A) List of lettuce Hsp60 genes. The

572 nomenclature of $L s H s p 60$ genes were assigned according to the closest AtHsp60 genes. (B)

573 Phylogenetic relationship of Hsp60 proteins of L. sativa and A. thaliana. LsHsp60 proteins are

574 marked in bold. LsHsp60 and AtHsp60 members in each subfamily (Cpn60, Cpn60 a, Cpn60ß,

575 and Hsp60) are represented in different colors. (C) Gene structure and motif composition

576 analysis of $\mathrm{LsHsp} 60$ genes. Blue boxes, green boxes, and black lines indicate untranslated region

577 (UTR), coding sequence (CDS), and intron, respectively. The numbers on each intron represent

578 the intron phase $(0,1$, or 2$)$. Detailed information of all motifs is shown in Additional file 3 :

579 Table S3.

580 Fig. 5. Phylogenetic analysis, exon-intron structure, and motif distribution of lettuce heat

581 shock protein 70 (LsHsp70) gene family. (A) List of Hsp70 genes in lettuce. Homologous

582 transcripts of lettuce Hsp70s were identified by BLASTP search using $A$. thaliana Hsp70 protein

583 sequences. The nomenclature of $L s H s p 70$ genes were assigned according to the name of

584 AtHsp70 gene of the highest similarity. (B) Phylogenetic relationship of Hsp70 proteins of $L$.

585 sativa and A. thaliana. LsHsp70 proteins are marked in bold. LsHsp70 and AtHsp70 members in

586 each subfamily (Hsp70, Hsp70t, mtHsc, cpHsc, BiP, and Hsp110/SSE) are represented in

587 different colors. 
588 Fig. 6. Gene structure and motif composition analysis of $L s H s p 70$ genes. (A) Blue boxes, green

589 boxes, and black lines indicate untranslated region (UTR), coding sequence (CDS), and intron,

590 respectively. The numbers on each intron represent the intron phase $(0,1$, or 2$)$. (B) Conserved

591 motif analysis of LsHsp70 proteins; 20 identified motifs are shown. Detailed information of all

592 motifs is shown in Additional file 3: Table S3.

593 Fig. 7. Characterization of lettuce heat shock protein 90 (LsHsp90) and heat shock protein 100

594 (LsHsp100) gene families. (A) Gene members of the Hsp90 family in lettuce (B). Phylogenetic

595 tree of Hsp90 proteins of L. sativa and A. thaliana. Lettuce Hsp proteins are marked in bold. (C)

596 Gene structure and motif composition analysis of LsHsp90. (D) Gene members of the LsHsp100

597 family. (E) Phylogenetic analysis of Hsp100 proteins of L. sativa and A. thaliana. (F). Gene

598 structure and motif composition analysis of $L s H s p 100$. Nomenclature of $L s H s p 90$ and $L s H s p 100$

599 genes were assigned according to the name of $A$. thaliana homologues. Lettuce Hsp90 and

600 Hsp100 family members are represented in different colors according to their subfamily (Group

601 Ia, Ib, Ila, and IIb in Hsp90ClpB-Cyt, ClpB-MT, ClpB-CP, ClpC, and ClpD in Hsp100. (C, F)

602 Exon-intron organization. Blue boxes, green boxes, and black lines indicate untranslated region

603 (UTR), coding sequence (CDS), and intron, respectively. The numbers on each intron represent

604 the intron phase $(0,1$, or 2$)$. Detailed information of all motifs is shown in Additional file 3 :

605 Table S3.

606 Fig. 8. Genome-wide distribution of lettuce triplicated regions highlighting chromosomal

607 location of Hsfs and Hsps genes. Lettuce syntenic regions are shown with colored blocks in 608 each chromosome (LG1 to LG9). Physical location of each LsHsf or LsHsp gene is shown and 609 gene names are colored according to their gene family. LsHsfC1a (Lsat_1_v5_gn_0_11820) was 
610 not included because it has not been mapped on any of the nine chromosomes in lettuce.

611 Boxes represent tandemly duplicated genes. Paralogous gene pairs originated from segmental

612 duplications are highlighted with circles and connected by lines.

613 Fig. 9. Cis-regulatory element analysis of lettuce Hsf and Hsp genes. Putative cis-regulatory

614 elements were analyzed in the $2 \mathrm{~kb}$ upstream regions of $L s H s f$ and $L s H s p$ genes. Six groups of

615 cis-regulatory elements (light response, stress response, hormone response, metabolism, cell

616 cycle, and circadian rhythm) are displayed. Colors from white to red indicate the occurrence of

617 each cis-regulatory element from 0 to $10+$ (10 or more).

618 Fig. 10. Gene expression analysis of lettuce Hsf and Hsp genes under different light

619 conditions. (A) Schematic representation of the experimental design used at different light

620 treatment on lettuce plants. L. sativa cultivar Codex plants were grown under light $150 \mu \mathrm{mol} \cdot \mathrm{m}^{-}$

$6212{ }^{2} \cdot \mathrm{s}^{-1}$ for 10 days then $220 \mu \mathrm{mol} \cdot \mathrm{m}^{-2} \cdot \mathrm{s}^{-1}$ was treated for 22 days. Control light $\left(\mu \mathrm{mol} \cdot \mathrm{m}^{-2} \cdot \mathrm{s}^{-1}\right), \mathrm{UV}$

622 light $\left(11 \mu \mathrm{mol} \cdot \mathrm{m}^{-2} \cdot \mathrm{s}^{-1}\right.$ supplemented with $220 \mu \mathrm{mol} \cdot \mathrm{m}^{-2} \cdot \mathrm{s}^{-1}$ of white light), and high light

$623\left(440 \mu \mathrm{mol} \cdot \mathrm{m}^{-2} \cdot \mathrm{s}^{-1}\right)$ were imposed to lettuce plants for 4 days. (B) Physical appearance of $L$.

624 sativa cultivar "Codex" 4 days after treatment of control light (CT), UV, and high light (HL). (C)

625 The relative expression of lettuce Hsf and Hsp genes. mRNA levels were determined by qRT-PCR

626 analysis and normalized to that of LsTUB. $\log _{2} \mathrm{FC}$ (fold change) values of relative expression of

627 treatment (UV and $\mathrm{HL}$ ) to the control (CT) are presented. Asterisks indicate significant

628 differences between treatment and control determined by Student's t test $(* p<0.05$,

$629 * * \mathrm{p}<0.01, * * * \mathrm{p}<0.001)$. Genes marked with the same letter (a-e) belong to the same

630 tandem duplicated gene cluster.

631 
Additional Table S1. General properties.

Additional Table S2. KaKs ratio

Additional Table S3. MEME motif

635 Additional Table S4. Tandem and segmental duplication

636 Additional Table S5. Cis-element analysis

637 Additional Table S6. Primers used for RT-qPCR analysis

\section{REFERENCES}

641 1. Resh HM. Hydroponic food production: a definitive guidebook for the advanced home 642 gardener and the commercial hydroponic grower. 7th ed. Boca Raton, FL: CRC Press; 2013.

643 2. Gómez C, Jiménez J. Effect of End-of-production High-energy Radiation on Nutritional 644 Quality of Indoor-grown Red-leaf Lettuce. horts. 2020;55:1055-60.

645 3. Zhou C, Zhang Y, Liu W, Zha L, Shao M, Li B. Light Quality Affected the Growth and Root 646 Organic Carbon and Autotoxin Secretions of Hydroponic Lettuce. Plants. 2020;9:1542.

647 4. Kim MJ, Moon Y, Tou JC, Mou B, Waterland NL. Nutritional value, bioactive compounds 648 and health benefits of lettuce (Lactuca sativa L.). Journal of Food Composition and Analysis. $6492016 ; 49: 19-34$.

650 5. Reyes-Chin-Wo S, Wang Z, Yang X, Kozik A, Arikit S, Song C, et al. Genome assembly with 651 in vitro proximity ligation data and whole-genome triplication in lettuce. Nat Commun.

$652 \quad 2017 ; 8: 14953$.

653 6. Bayat L, Arab M, Aliniaeifard S, Seif M, Lastochkina O, Li T. Effects of growth under 654 different light spectra on the subsequent high light tolerance in rose plants. AoB PLANTS. 655 2018;10. doi:10.1093/aobpla/ply052.

656 7. Silvestri C, Caceres ME, Ceccarelli M, Pica AL, Rugini E, Cristofori V. Influence of 657 Continuous Spectrum Light on Morphological Traits and Leaf Anatomy of Hazelnut Plantlets. 658 Front Plant Sci. 2019;10:1318.

659 8. Huang J, Zhao X, Chory J. The Arabidopsis Transcriptome Responds Specifically and 660 Dynamically to High Light Stress. Cell Reports. 2019;29:4186-4199.e3. 
9. Crisp PA, Ganguly DR, Smith AB, Murray KD, Estavillo GM, Searle I, et al. Rapid Recovery Gene Downregulation during Excess-Light Stress and Recovery in Arabidopsis. Plant Cell. 2017;29:1836-63.

10. Swindell WR, Huebner M, Weber AP. Transcriptional profiling of Arabidopsis heat shock proteins and transcription factors reveals extensive overlap between heat and non-heat stress response pathways. BMC Genomics. 2007;8:125.

11. Hu W, Hu G, Han B. Genome-wide survey and expression profiling of heat shock proteins and heat shock factors revealed overlapped and stress specific response under abiotic stresses in rice. Plant Science. 2009;176:583-90.

12. Zhang J, Liu B, Li J, Zhang L, Wang Y, Zheng H, et al. Hsf and Hsp gene families in Populus: genome-wide identification, organization and correlated expression during development and in stress responses. BMC Genomics. 2015;16:181.

13. Yang X, Zhu W, Zhang H, Liu N, Tian S. Heat shock factors in tomatoes: genome-wide identification, phylogenetic analysis and expression profiling under development and heat stress. PeerJ. 2016;4:e1961.

14. Begcy K, Weigert A, Egesa A, Dresselhaus T. Compared to Australian Cultivars, European Summer Wheat (Triticum aestivum) Overreacts When Moderate Heat Stress Is Applied at the Pollen Development Stage. Agronomy. 2018;8:99.

15. Kryazhimskiy S, Plotkin JB. The Population Genetics of dN/dS. PLoS Genet. 2008;4:e1000304.

16. Nielsen $\mathrm{H}$, Wernersson R. An overabundance of phase 0 introns immediately after the start codon in eukaryotic genes. BMC Genomics. 2006;7:256.

17. Rowarth NM, Dauphinee AN, Denbigh GL, Gunawardena AH. Hsp70 plays a role in programmed cell death during the remodelling of leaves of the lace plant (Aponogeton madagascariensis). Journal of Experimental Botany. 2019;:erz447.

18. Herath V, Gayral M, Adhikari N, Miller R, Verchot J. Genome-wide identification and characterization of Solanum tuberosum BiP genes reveal the role of the promoter architecture in BiP gene diversity. Sci Rep. 2020;10:11327.

19. Zhang J, Li J, Liu B, Zhang L, Chen J, Lu M. Genome-wide analysis of the Populus Hsp90 gene family reveals differential expression patterns, localization, and heat stress responses. BMC Genomics. 2013;14:532.

20. Guo M, Liu J-H, Ma X, Luo D-X, Gong Z-H, Lu M-H. The Plant Heat Stress Transcription Factors (HSFs): Structure, Regulation, and Function in Response to Abiotic Stresses. Front Plant Sci. 2016;7. doi:10.3389/fpls.2016.00114.

21. Hahn A, Bublak D, Schleiff E, Scharf K-D. Crosstalk between Hsp90 and Hsp70 Chaperones and Heat Stress Transcription Factors in Tomato. Plant Cell. 2011;23:741-55. 
22. Zhu J-K. Abiotic Stress Signaling and Responses in Plants. Cell. 2016;167:313-24.

698 23. Begcy K, Dresselhaus T. Epigenetic responses to abiotic stresses during reproductive 699 development in cereals. Plant Reprod. 2018;31:343-355.

700 24. Rejeb I, Pastor V, Mauch-Mani B. Plant Responses to Simultaneous Biotic and Abiotic 701 Stress: Molecular Mechanisms. Plants. 2014;3:458-75.

702 25. Lin Y-X, Jiang H-Y, Chu Z-X, Tang X-L, Zhu S-W, Cheng B-J. Genome-wide identification, classification and analysis of heat shock transcription factor family in maize. BMC 704 Genomics. 2011;12:76.

26. Wang C, Zhang Q, Shou H. Identification and expression analysis of OsHsfs in rice. J Zhejiang Univ Sci B. 2009;10:291-300.

27. Scharf K-D, Berberich T, Ebersberger I, Nover L. The plant heat stress transcription factor (Hsf) family: Structure, function and evolution. Biochimica et Biophysica Acta (BBA) - Gene 709 Regulatory Mechanisms. 2012;1819:104-19.

710 28. Li H, Yan S, Zhao L, Tan J, Zhang Q, Gao F, et al. Histone acetylation associated up711 regulation of the cell wall related genes is involved in salt stress induced maize root swelling. 712 BMC Plant Biol. 2014;14:105.

713 29. Yoshida T, Ohama N, Nakajima J, Kidokoro S, Mizoi J, Nakashima K, et al. Arabidopsis HsfA1 transcription factors function as the main positive regulators in heat shock-responsive gene expression. Mol Genet Genomics. 2011;286:321-32.

30. Albihlal WS, Obomighie I, Blein T, Persad R, Chernukhin I, Crespi M, et al. Arabidopsis HEAT SHOCK TRANSCRIPTION FACTORA1b regulates multiple developmental genes under benign and stress conditions. Journal of Experimental Botany. 2018;69:2847-62.

721 32. Cannon SB, Mitra A, Baumgarten A, Young ND, May G. [No title found]. BMC Plant Biol. $722 \quad 2004 ; 4: 10$.

723 33. Xu G, Guo C, Shan H, Kong H. Divergence of duplicate genes in exon-intron structure.

724 Proceedings of the National Academy of Sciences. 2012;109:1187-92.

34. Son K-H, Oh M-M. Growth, photosynthetic and antioxidant parameters of two lettuce cultivars as affected by red, green, and blue light-emitting diodes. Hortic Environ Biotechnol. 2015;56:639-53. 2018;6:e4607. 
36. Jung H-S, Crisp PA, Estavillo GM, Cole B, Hong F, Mockler TC, et al. Subset of heat-shock transcription factors required for the early response of Arabidopsis to excess light. Proceedings of the National Academy of Sciences. 2013;110:14474-9.

37. Yamamoto Y. Quality Control of Photosystem II: The Mechanisms for Avoidance and Tolerance of Light and Heat Stresses are Closely Linked to Membrane Fluidity of the Thylakoids. Front Plant Sci. 2016;7. doi:10.3389/fpls.2016.01136.

38. Goodstein DM, Shu S, Howson R, Neupane R, Hayes RD, Fazo J, et al. Phytozome: a comparative platform for green plant genomics. Nucleic Acids Research. 2012;40:D1178-86.

39. Le SQ, Gascuel O. An Improved General Amino Acid Replacement Matrix. Molecular Biology and Evolution. 2008;25:1307-20.

40. Stecher G, Tamura K, Kumar S. Molecular Evolutionary Genetics Analysis (MEGA) for macOS. Molecular Biology and Evolution. 2020;37:1237-9.

41. Begcy K, Mariano ED, Lembke CG, Zingaretti SM, Souza GM, Araújo P, et al. Overexpression of an evolutionarily conserved drought-responsive sugarcane gene enhances salinity and drought resilience. Annals of Botany. 2019;124:691-700.

42. Gasteiger E, Hoogland C, Gattiker A, Duvaud S, Wilkins MR, Appel RD, et al. Protein Identification and Analysis Tools on the ExPASy Server. In: Walker JM, editor. The Proteomics Protocols Handbook. Totowa, NJ: Humana Press; 2005. p. 571-607. doi:10.1385/1-59259-890$0: 571$.

43. Horton P, Park K-J, Obayashi T, Fujita N, Harada H, Adams-Collier CJ, et al. WoLF PSORT: protein localization predictor. Nucleic Acids Research. 2007;35 Web Server:W585-7.

44. Edgar RC. MUSCLE: multiple sequence alignment with high accuracy and high throughput. Nucleic Acids Research. 2004;32:1792-7.

45. Smith TF, Waterman MS. Identification of common molecular subsequences. Journal of Molecular Biology. 1981;147:195-7.

46. Suyama M, Torrents D, Bork P. PAL2NAL: robust conversion of protein sequence alignments into the corresponding codon alignments. Nucleic Acids Research. 2006;34 Web Server:W609-12.

47. Hu B, Jin J, Guo A-Y, Zhang H, Luo J, Gao G. GSDS 2.0: an upgraded gene feature visualization server. Bioinformatics. 2015;31:1296-7.

48. Bailey TL, Boden M, Buske FA, Frith M, Grant CE, Clementi L, et al. MEME SUITE: tools for motif discovery and searching. Nucleic Acids Research. 2009;37 Web Server:W202-8.

49. Lescot M. PlantCARE, a database of plant cis-acting regulatory elements and a portal to tools for in silico analysis of promoter sequences. Nucleic Acids Research. 2002;30:325-7. 
765 50. Begcy K, Dresselhaus T. Tracking maize pollen development by the Leaf Collar Method. 766 Plant Reprod. 2017;30:171-8.

767 51. Begcy K, Nosenko T, Zhou L-Z, Fragner L, Weckwerth W, Dresselhaus T. Male Sterility in 768 Maize after Transient Heat Stress during the Tetrad Stage of Pollen Development. Plant Physiol. $7692019 ; 181: 683-700$.

770 52. Livak KJ, Schmittgen TD. Analysis of Relative Gene Expression Data Using Real-Time 771 Quantitative PCR and the 2- $\Delta \Delta$ CT Method. Methods. 2001;25:402-8. 
Figures

A

\begin{tabular}{|ccccc|}
\hline & L. sativa & A. thaliana & P. trichocarpa & O. sativa \\
\hline Hsf & 32 & 21 & 28 & 25 \\
\hline sHsp & 32 & 19 & 37 & 29 \\
\hline Hsp60 & 22 & 18 & 28 & 20 \\
\hline Hsp70 & 64 & 18 & 20 & 27 \\
\hline Hsp90 & 7 & 7 & 10 & 8 \\
\hline Hsp100 & 8 & 7 & 5 & 9 \\
\hline
\end{tabular}

C

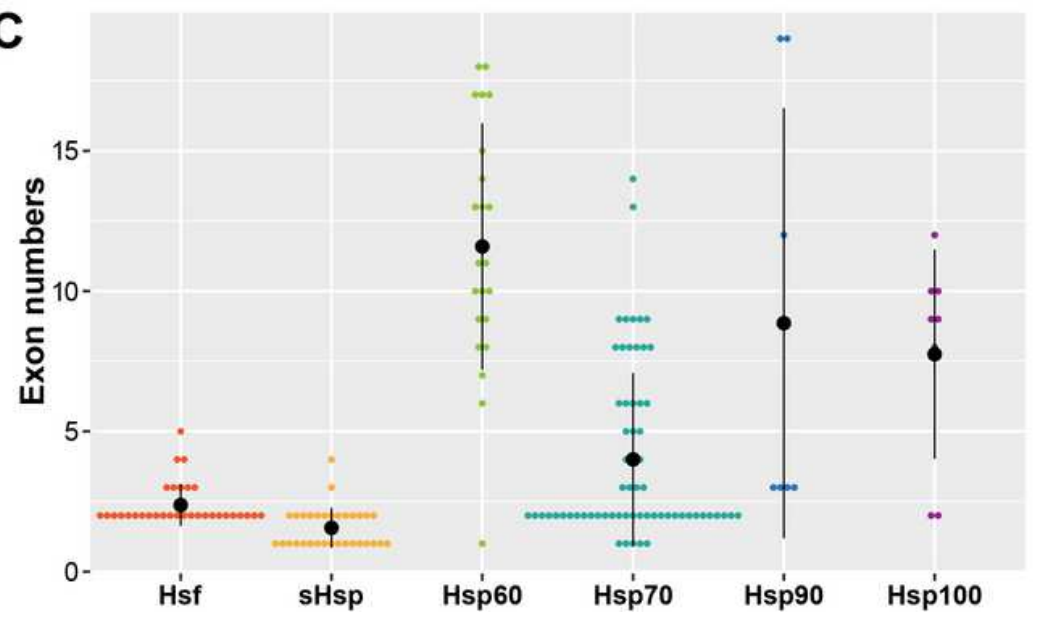

B

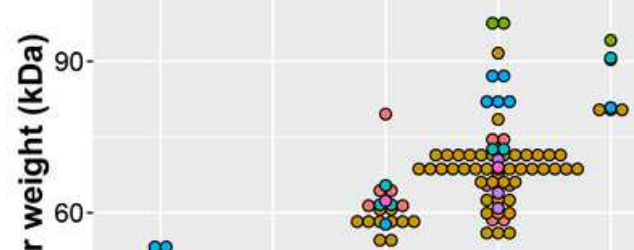

ocalization

- chloroplast

- cytoplasm

- E.R.

- extracellular

- mitochondria

- nucleus

- peroxisome

- plasma membrane

D 0.4 -

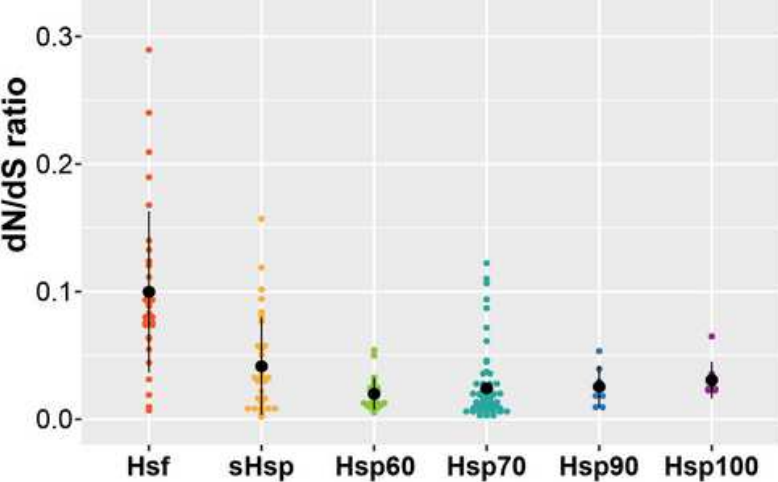

\section{Figure 1}

General gene structure features of lettuce Hsfs and Hsps. (A) Numbers of Hsf and Hsp genes in lettuce, Arabidopsis, poplar and rice. (B) Molecular weight analysis of LsHsfs and LsHsps. (C) Exon number distribution of the lettuce Hsfs and Hsps. (D) Evaluation of positive selection of LsHsfs and LsHsps families based on the $\mathrm{dN} / \mathrm{dS}$ ratio analysis. 
A

\begin{tabular}{|c|c|c|}
\hline Transcript & Gene name & Subfamily \\
\hline Lsat_1_v5_gn_3_19100.1 & LsHsfA1a & \multirow{13}{*}{ Type A } \\
\hline Lsat_1_v5_gn_6_1620.1 & LsHsfA1b & \\
\hline Lsat_1_v5_gn_5_48320.1 & LsHsfA2 & \\
\hline Lsat_1_v5_gn_1_50141.1 & LsHsfA3 & \\
\hline Lsat_1_v5_gn_4_26761.1 & LsHsfA4a & \\
\hline Lsat_1_v5_gn_4_48361.1 & LsHsfA4b & \\
\hline Lsat_1_v5_gn_7_25320.2 & LsHsfA4c & \\
\hline Lsat_1_v5_gn_1_70440.1 & LsHsfA5 & \\
\hline Lsat_1_v5_gn_5_142940.1 & LsHsfA6 & \\
\hline Lsat_1_v5_gn_5_185701.1 & LsHsfA7a & \\
\hline Lsat_1_v5_gn_8_55640,3 & LsHsfA7b & \\
\hline Lsat_1_v5_gn_9_7580.1 & LsHsfA7c & \\
\hline Lsat_1_v5_gn_8_35220.1 & LsHsfA8 & \\
\hline Lsat_1_v5_gn_3_90920.1 & LsHsfB1a & \multirow{12}{*}{ Type B } \\
\hline Lsat_1_v5_gn_4_165921.1 & LsHsfB1b & \\
\hline Lsat_1_v5_gn_2_133880.1 & LsHsfB2a & \\
\hline Lsat_1_v5_gn_3_8400.1 & LsHsfB2b & \\
\hline Lsat_1_v5_gn_8_6800.1 & LsHsfB2c & \\
\hline Lsat_1_v5_gn_4_52841.1 & LsHsfB3a & \\
\hline Lsat_1_v5_gn_9_30661.1 & LsHsfB3b & \\
\hline Lsat_1_v5_gn_1_21321.1 & LsHsfB4a & \\
\hline Lsat_1_v5_gn_4_126681.2 & LsHsfB $4 b$ & \\
\hline Lsat_1_v5_gn_1_126880.2 & LsHsfB5a & \\
\hline Lsat_1_v5_gn_7_19541.1 & LsHsfB5b & \\
\hline Lsat_1_v5_gn_7_19561.1 & LsHsfB5c & \\
\hline Lsat_1_v5_gn_0_11820.1 & LsHsfC1a & \multirow{7}{*}{ Type C } \\
\hline Lsat_1_v5_gn_4_81660.1 & LsHsfC1b & \\
\hline Lsat_1_v5_gn_5_103200.1 & LsHsfC1c & \\
\hline Lsat_1_v5_gn_5_172781.1 & LsHsfC1d & \\
\hline Lsat_1_v5_gn_6_77581.1 & LsHsfC1e & \\
\hline Lsat_1_v5_gn_7_40921.1 & LsHsfC1f & \\
\hline Lsat_1_v5_gn_8_105120.1 & LsHsfC1g & \\
\hline
\end{tabular}

C

LsHsfA7a

LsHsfA7c

LsHsfA2

LsHsfA6

LsHsfA1a

LsHsfA1b

LsHsfA8

LsHsfA5

LsHsfA4a

LsHsfA4b

LsHsfA4c

LsHsfA3

LsHsfB 5 b

LsHsfB5c

LsHsfB5a

LsHsfB3b

LsHsfB3a

LsHsfB2a

LsHsfB2C

LsHsfB2b

LsHsfB1a

sHsfB1b

LsHsfB 4a

LsHsfB4b

LsHsfC1c

LsHsfC1g

LsHsfC1f

LsHsfC1a

LsHsfC1d

LsHsfC1b

LsHsfC1e

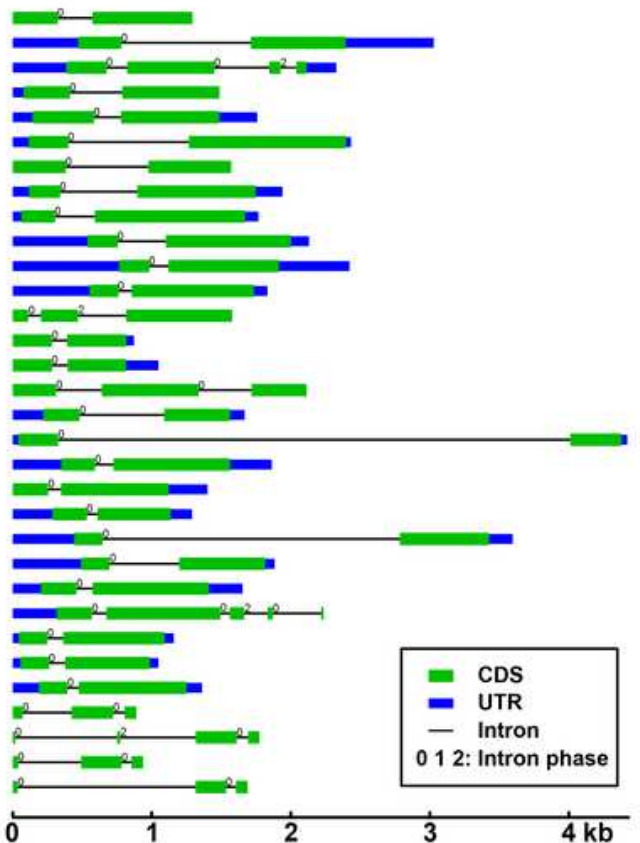

B
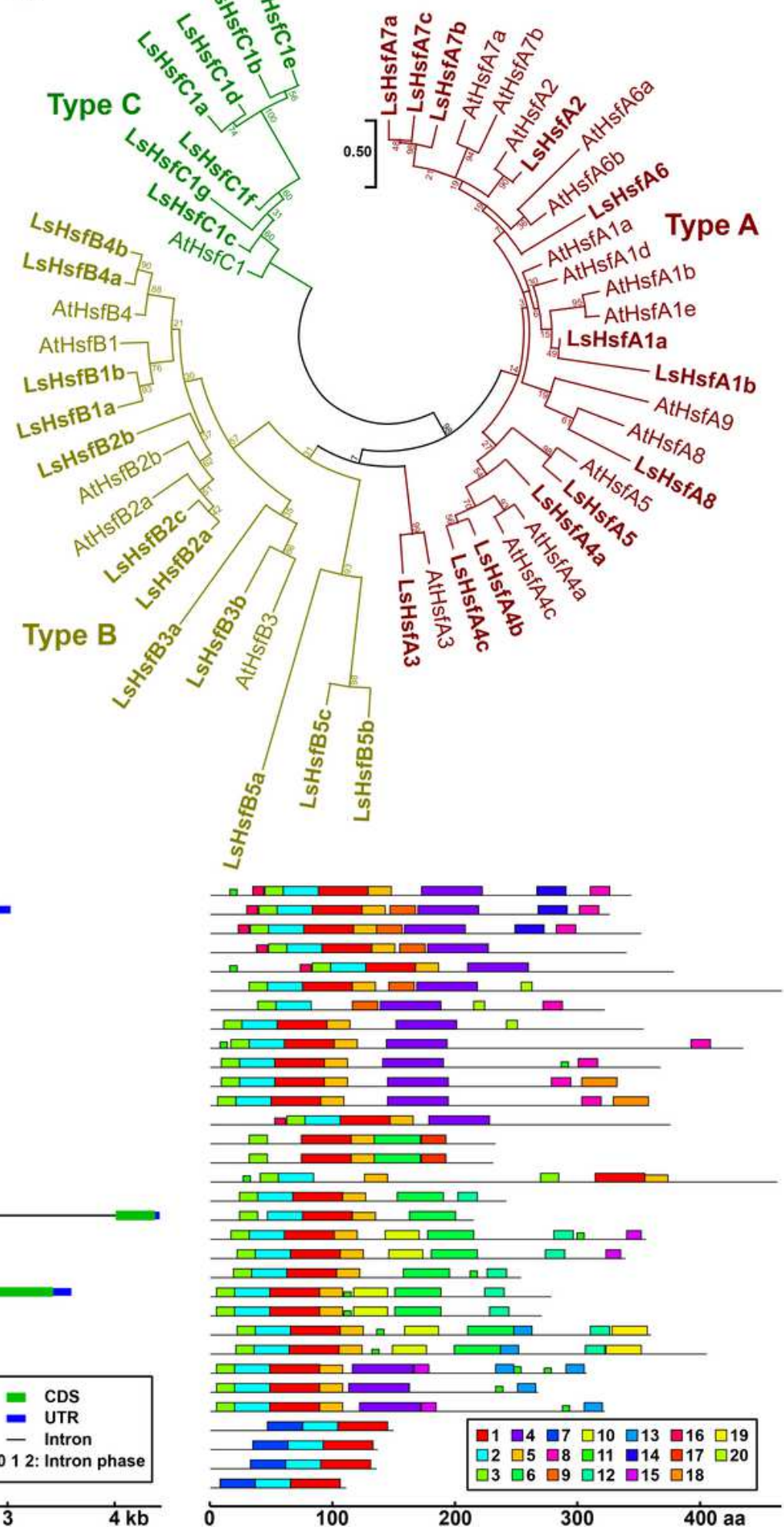

Figure 2

Phylogenetic analysis, exon-intron structure, and motif distribution of lettuce heat shock transcription factor (LsHsf) gene family. (A) List of lettuce Hsf genes. (B) Phylogenetic relationship of Hsf proteins of lettuce and Arabidopsis. LsHsf and AtHsf members in each subfamily (Type A, Type B, and Type C) are represented in different colors. (C) Gene structure and motif composition analysis of LsHsf genes with blue boxes, green boxes, and black lines indicate untranslated region (UTR), coding sequence (CDS), and 
intron, respectively. The numbers on each intron represent the intron phase $(0,1$, or 2$)$. Detailed information of all motifs is shown in Additional file 3: Table S3.

\section{A}

\begin{tabular}{|c|c|c|}
\hline Transcript & Gene name & Subfamily \\
\hline Lsat_1_v5_gn_2_28040.1 & LsHsp11.8-Cl & \multirow{12}{*}{$\mathrm{sHsp} \cdot \mathrm{Cl}$} \\
\hline Lsat_1_v5_gn_5_171121.1 & LsHsp17.4-Cl & \\
\hline Lsat_1_v5_gn_8_72460.1 & LsHsp17.6-Cl & \\
\hline Lsat_1_v5_gn_8_72420.1 & LsHsp17.7-Cl & \\
\hline Lsat_1_v5_gn_5_168220.5 & LsHsp17.8-Cl & \\
\hline Lsat_1_v5_gn_2_120060.1 & LsHsp17.9-CI & \\
\hline Lsat_1_v5_gn_2_28100.1 & LsHsp18.0-Cl & \\
\hline Lsat_1_v5_gn_2_28120.2 & LsHsp $18.4 \cdot \mathrm{Cl}$ & \\
\hline Lsat_1_v5_gn_2_120081.1 & LsHsp18.5-Cl & \\
\hline Lsat_1_v5_gn_2_28080.3 & LsHsp18.9-Cl & \\
\hline Lsat_1_v5_gn_2_120040.1 & LsHsp20.6-Cl & \\
\hline Lsat_1_v5_gn_2_28000.1 & LsHsp23.8-Cl & \\
\hline Lsat_1_v5_gn_7_61081.1 & LsHsp18.2-CII & \multirow{6}{*}{ sHsp-CII } \\
\hline Lsat_1_v5_gn_7_61061.1 & LsHsp18.3A-CII & \\
\hline Lsat_1_v5_gn_7_61041.2 & LsHsp18.3B-CII & \\
\hline Lsat_1_v5_gn_7_60201.1 & LsHsp18.4A-CII & \\
\hline Lsat_1_v5_gn_7_61001.1 & LsHsp18.48-CII & \\
\hline Lsat_1_v5_gn_7_60240.1 & LsHsp19.8-CII & \\
\hline Lsat_1_v5_gn_5_136741.1 & LsHsp17.8-CIII & \multirow{2}{*}{ sHsp-CIII } \\
\hline Lsat_1_v5_gn_2_123840,3 & LsHsp20.7-CIII & \\
\hline Lsat_1_v5_gn_7_17661.1 & LsHsp15.6-CIV & \multirow{2}{*}{ sHsp-CIV } \\
\hline Lsat_1_v5_gn_4_59740.2 & LsHsp19.2-CIV & \\
\hline Lsat_1_v5_gn_7_108120.1 & LsHsp22.0-CV & sHsp.CV \\
\hline Lsat_1_v5_gn_9_81920.1 & LsHsp21.8-ER & \multirow{3}{*}{ sHsp-ER } \\
\hline Lsat_1_v5_gn_8_130841.1 & LsHsp23.2-ER & \\
\hline Lsat_1_v5_gn_9_82001.1 & LsHsp37 6-ER & \\
\hline Lsat_1_v5_gn_3_481.1 & LsHsp24.4-M & \multirow{3}{*}{ sHsp-MT } \\
\hline Lsat_1_v5_gn_6_91340.1 & LsHsp24.6-M & \\
\hline Lsat_1_v5_gn_1_33281.3 & LsHsp25.1-M & \\
\hline Lsat_1_v5_gn_8_4541.1 & LsHsp26.1.P & \multirow{2}{*}{ sHsp-CP } \\
\hline Lsat_1_v5_gn_8_151001.1 & LsHsp26.6-P & \\
\hline Lsat_1_v5_gn_3_65081.1 & LsHsp15.6-PX & sHsp-PX \\
\hline
\end{tabular}

C

B
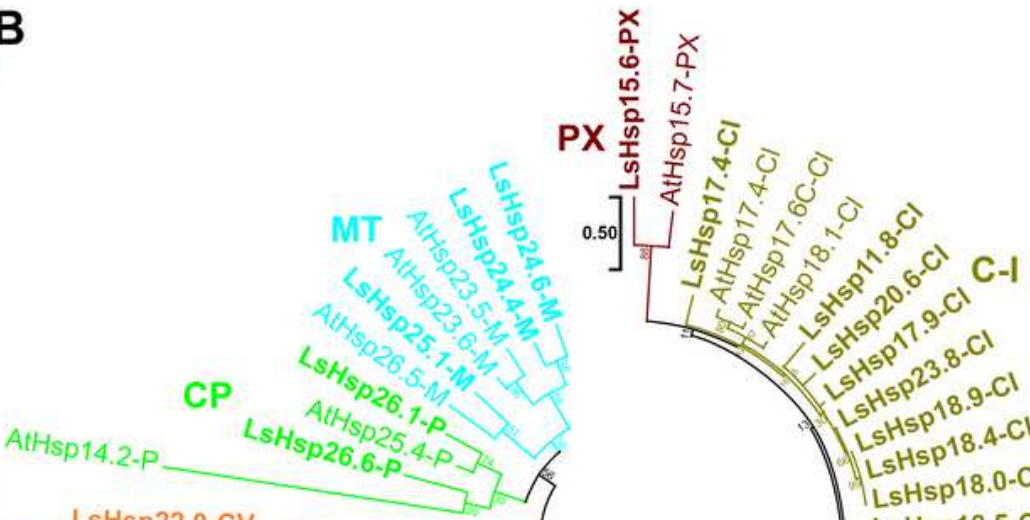

C-V

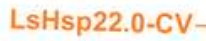
AtHsp2 17

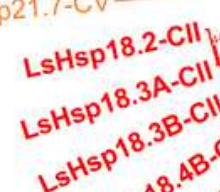

\section{C}

LsHsp15.6-PX LsHsp17.4-CI

LsHsp11.8-Cl

LsH

LsHsp23.8-CI

s s isp18.9-Cl

LsHsp18.4-C

LsHsp18.0-C

LsHsp18.5-C

LsHsp17.8-CI

LsHsp17.7-C

LsHsp17.6-CI

LsHsp23.2-ER

LsHsp21.8-ER

LsHsp37.6-ER

LsHsp19.2-CIV

LsHsp15.6-CIV

LsHsp20.7-CII

LsHsp17.8-CIII

sHsp19.8-CII

LsHsp18.4A-CII

LsHsp18.4B-CI

LsHsp18.3B-CII

LsHsp18.3A-CI

LsHsp18.2-CII

LsHsp22.0-CV

LsHsp26.6-P

LsHsp26.1-P

LsHsp25.1-M

-sHsp24.4-M

LsHsp24.6-M

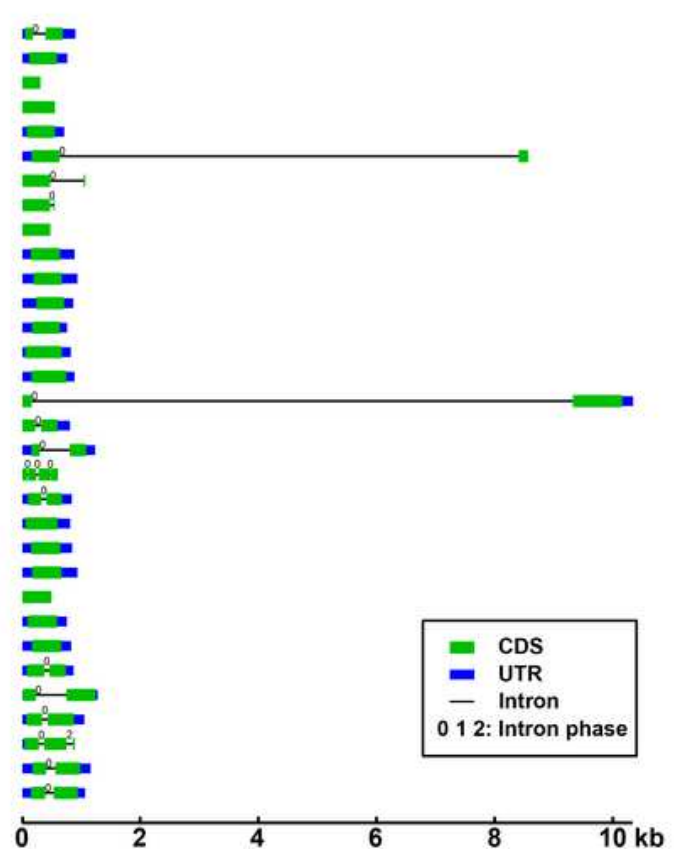

Figure 3

Identification, phylogenetic and gene structure analysis of lettuce small heat shock protein (LssHsp) gene family. (A) List of sHsp genes in lettuce. Classification of LssHsp genes were assigned according to their molecular weight and subcellular localization (Additional Table S1). (B) Phylogenetic relationship of 
LsHsp proteins and A. thaliana. LssHsp proteins are marked in bold. LssHsp and AtsHsp members in each subfamily (C-I, C-II, C-III, C-IV, C-V, C-VI, ER, MT, CP, and PX) are represented in different colors. (C) Gene structure and motif composition analysis of LssHsp genes. Blue boxes, green boxes, and black lines indicate untranslated region (UTR), coding sequence (CDS), and intron, respectively. The numbers on each intron represent the intron phase $(0,1$, or 2$)$. Detailed information of all motifs is shown in Additional file 3: Table S3.

\begin{tabular}{|c|c|c|}
\hline Transcript & Gene name & Subfamily \\
\hline Lsat_1_v5_gn_4_42920.1 & LsCpn60-1 & \multirow{12}{*}{ Cpn60 } \\
\hline Lsat_1_v5_gn_4_153000.1 & LsCpn60-2 & \\
\hline Lsat_1_v5_gn_1_55661.1 & LsCpn60-4a & \\
\hline Lsat_1_v5_gn_6_31340.1 & LsCpn60-4b & \\
\hline Lsat_1_v5_gn_4_22680.1 & LsCpn60-5a & \\
\hline Lsat_1_v5_gn_8_34780.1 & LsCpn60-5b & \\
\hline Lsat_1_v5_gn_5_64940.1 & LsCpn60-6 & \\
\hline Lsat_1_v5_gn_3_23880.1 & LsCpn60-7a & \\
\hline Lsat_1_v5_gn_5_47521.1 & LsCpn60-7b & \\
\hline Lsat_1_v5_gn_5_156500.1 & LsCpn60-8 & \\
\hline Lsat_1_v5_gn_4_2661.1 & LsCpn60-9a & \\
\hline Lsat_1_v5_gn_8_155141.1 & LsCpn60-9b & \\
\hline Lsat_1_v5_gn_1_63701.1 & LsCpn60a1 & \multirow{4}{*}{ Cpn60a } \\
\hline Lsat_1_v5_gn_2_63681.1 & LsCpn60a2 & \\
\hline Lsat_1_v5_gn_2_99661.1 & LsCpn60a3 & \\
\hline Lsat_1_v5_gn_2_99701.1 & LsCpn60a4 & \\
\hline Lsat_1_v5_gn_2_50361.1 & LsCpn60ß1 & \multirow{3}{*}{ Cpn60 } \\
\hline Lsat_1_v5_gn_5_134540.1 & LsCpn60ß2 & \\
\hline Lsat_1_v5_gn_8_37600.1 & LsCpn60ß3 & \\
\hline Lsat_1_v5_gn_9_11221.1 & LsHsp60-1 & \multirow{3}{*}{ Hsp60 } \\
\hline Lsat_1_v5_gn_6_63121.1 & LsHsp60-2 & \\
\hline Lsat_ 1 v5 gn_1 35540.1 & LsHsp60-3 & \\
\hline
\end{tabular}

\section{B}

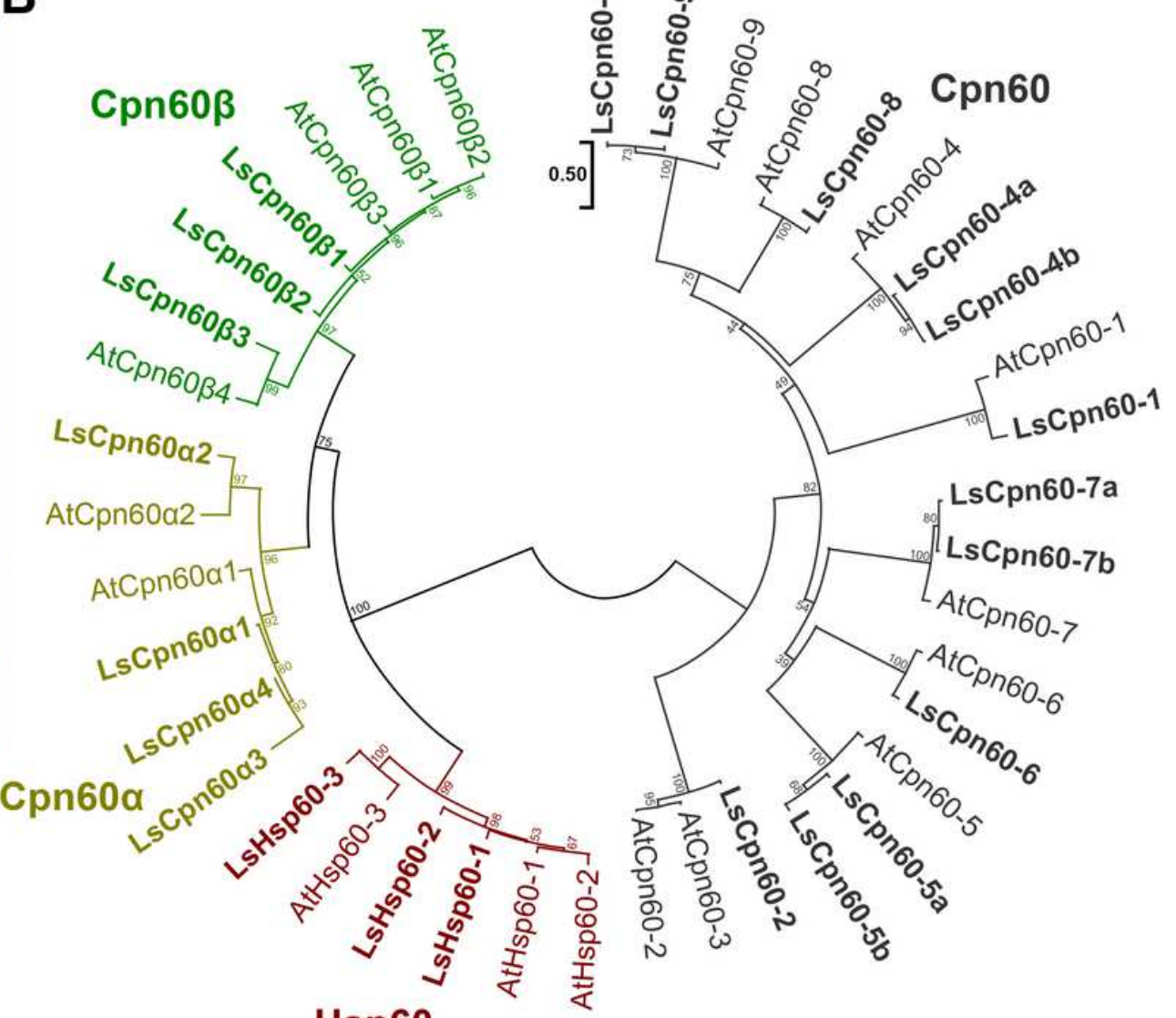

C

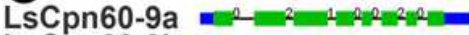

LsCpn60-9b 들

LsCpn60-8

LsCpn60-4a

LsCpn60-4b

LsCpn60-1

LsCpn60-7a

LsCpn60-7b

LsCpn60-6

LsCpn60-5a

LsCpn60-5b

LsCpn60-2

LsHsp60-1

LsHsp60-2

LsHsp60-3

LsCpn60a3

LsCpn60a4

LsCpn60a1

LsCpn60a2

LsCpn60ß3

LsCpn60ß2

LsCpn60 $\beta 1$

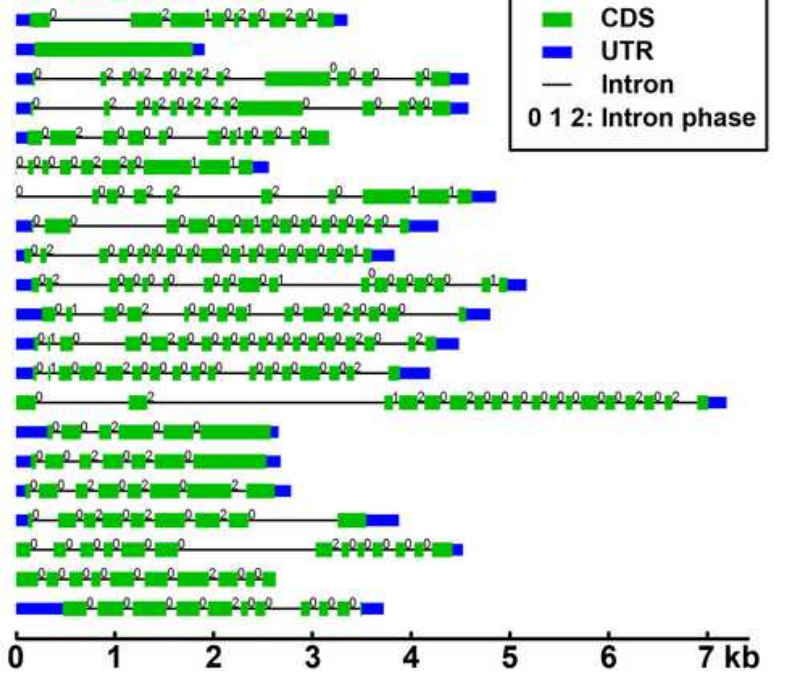

\section{Hsp60}

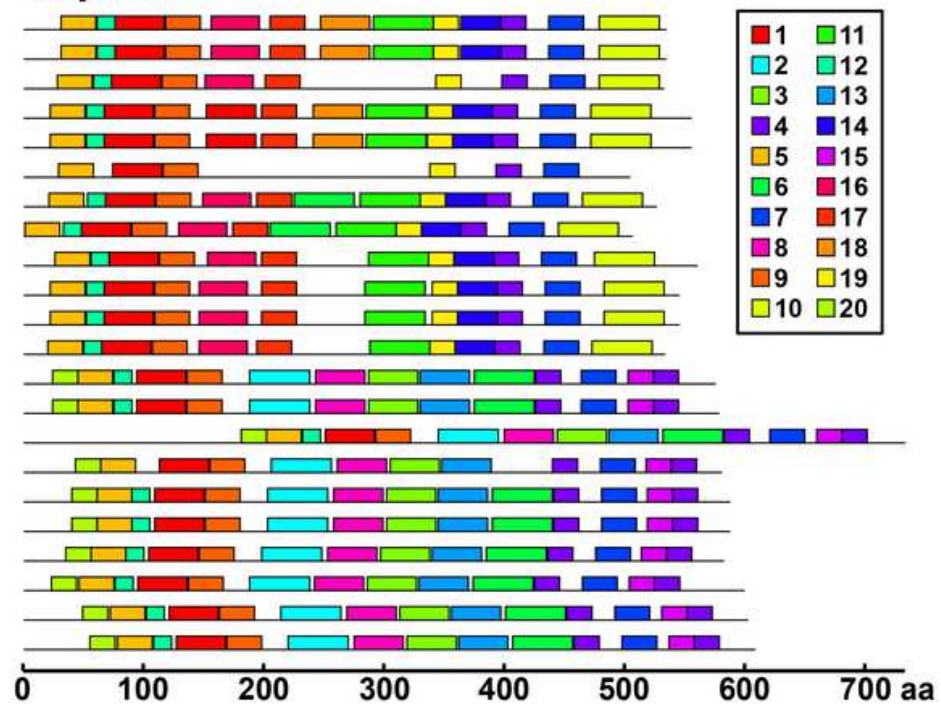

Figure 4 
Classification, evolutionary relationship, gene structure and motif distribution of lettuce heat shock protein 60 (LsHsp60) gene family. (A) List of lettuce Hsp60 genes. The nomenclature of LsHsp60 genes were assigned according to the closest AtHsp60 genes. (B) Phylogenetic relationship of Hsp60 proteins of L. sativa and A. thaliana. LsHsp60 proteins are marked in bold. LsHsp60 and AtHsp60 members in each subfamily (Cpn60, Cpn60a, Cpn60ß, and Hsp60) are represented in different colors. (C) Gene structure and motif composition analysis of LsHsp60 genes. Blue boxes, green boxes, and black lines indicate untranslated region (UTR), coding sequence (CDS), and intron, respectively. The numbers on each intron represent the intron phase $(0,1$, or 2$)$. Detailed information of all motifs is shown in Additional file 3: Table S3.

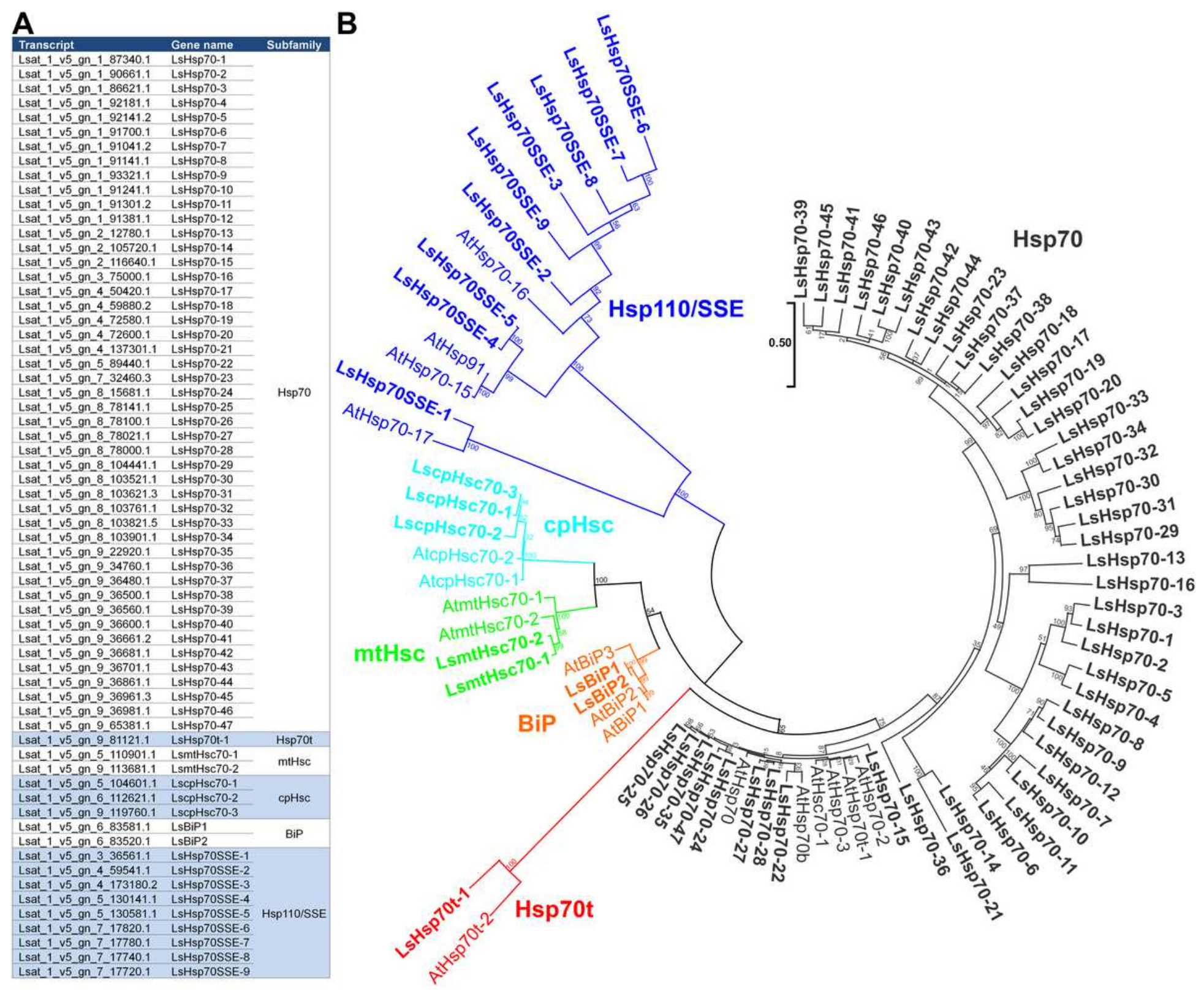

Figure 5

Phylogenetic analysis, exon-intron structure, and motif distribution of lettuce heat shock protein 70 (LsHsp70) gene family. (A) List of Hsp70 genes in lettuce. Homologous transcripts of lettuce Hsp70s were identified by BLASTP search using A. thaliana Hsp70 protein sequences. The nomenclature of 
LsHsp70 genes were assigned according to the name of AtHsp70 gene of the highest similarity. (B) Phylogenetic relationship of Hsp70 proteins of L. sativa and A. thaliana. LsHsp70 proteins are marked in bold. LsHsp70 and AtHsp70 members in each subfamily (Hsp70, Hsp70t, mtHsc, cpHsc, BiP, and Hsp110/SSE) are represented in different colors.

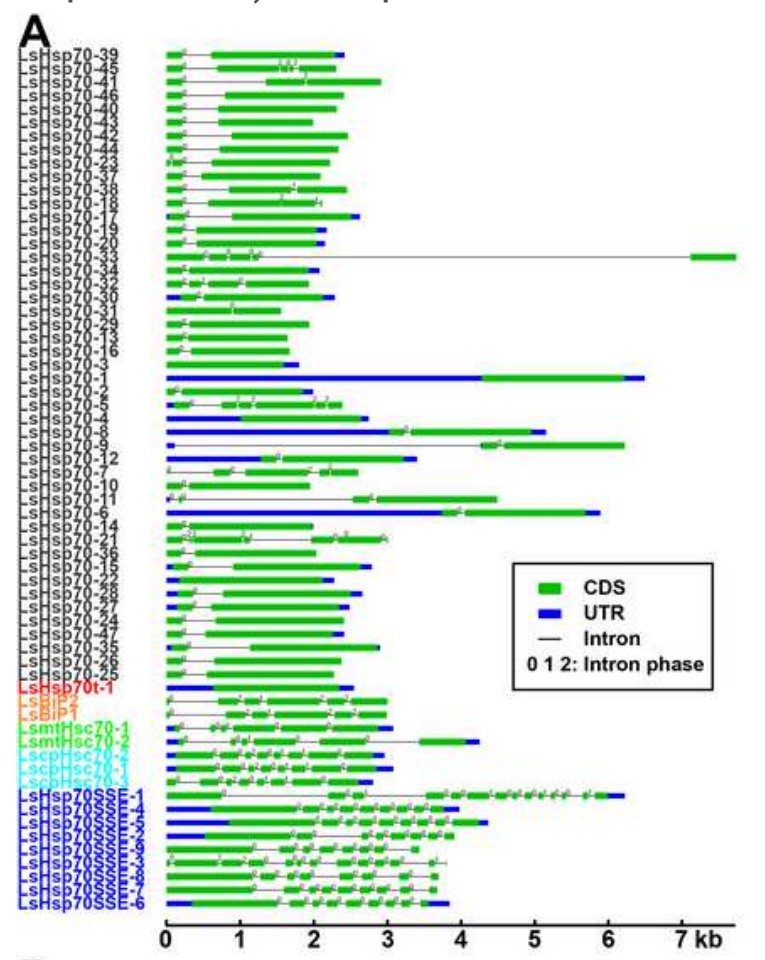

B

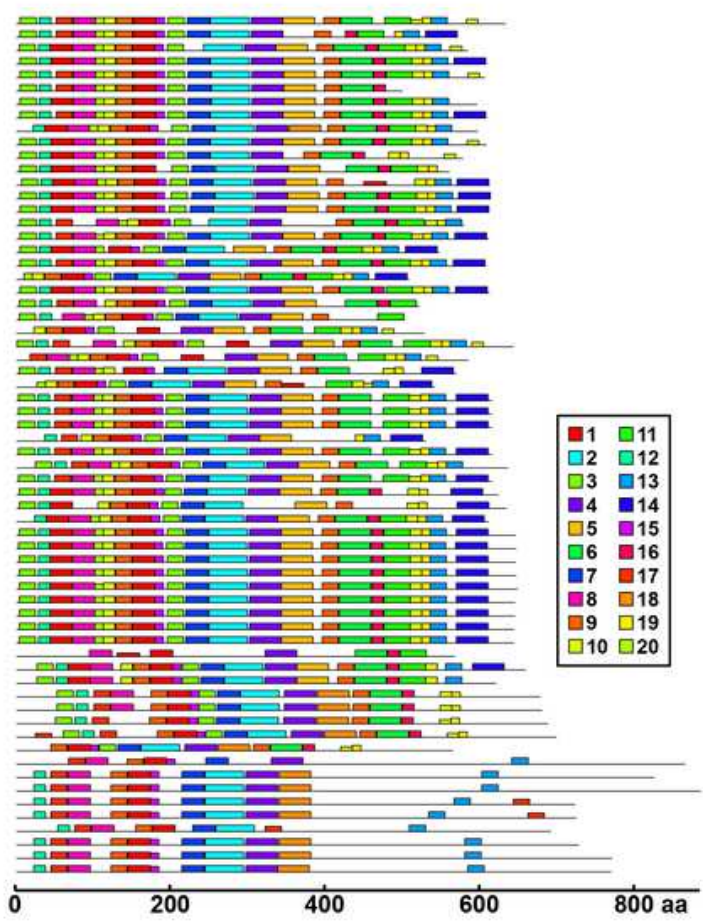

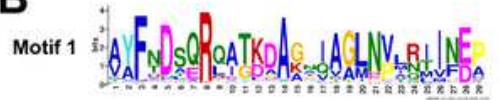

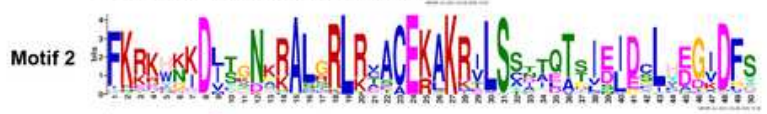

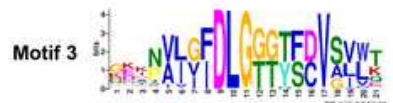

Motif 4 ind

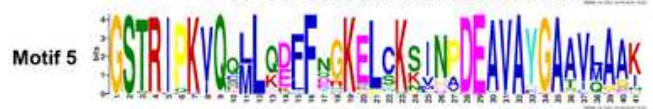

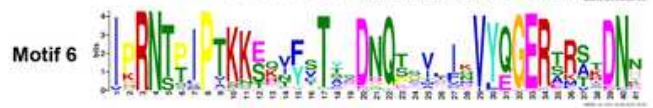

war if

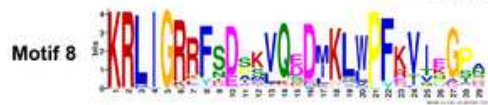

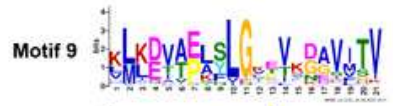

Motif 10 iै

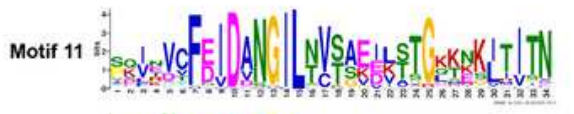

Motif 12 iz

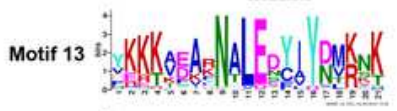

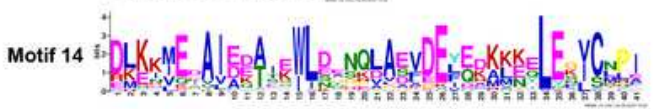

Motif 15 :

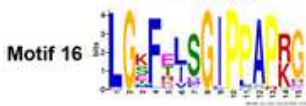

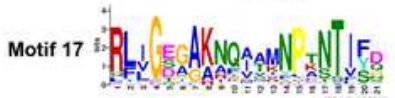

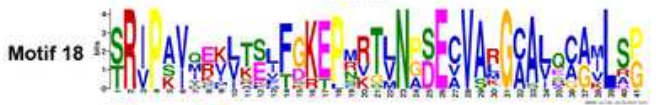

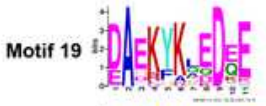

Motif 20 in

Figure 6 
Gene structure and motif composition analysis of LsHsp70 genes. (A) Blue boxes, green boxes, and black lines indicate untranslated region (UTR), coding sequence (CDS), and intron, respectively. The numbers on each intron represent the intron phase $(0,1$, or 2$)$. (B) Conserved motif analysis of LsHsp70 proteins; 20 identified motifs are shown. Detailed information of all motifs is shown in Additional file 3: Table S3.

\begin{tabular}{|lll|} 
A & & \\
\hline Transcript & Gene name & Subfamily \\
\hline Lsat_1_v5_gn_2_131360.1 & LsHsp90-1a & \multirow{2}{*}{ Group la } \\
\hline Lsat_1_v5_gn_8_6561.1 & LsHsp90-1b & \\
\hline Lsat_1_v5_gn_9_89140.1 & LsHsp90-2a & \multirow{2}{*}{ Group Ib } \\
\hline Lsat_1_V5_gn_9_89121.1 & LsHsp90-2b & \\
\hline Lsat_1_v5_gn_4_20860.1 & LsHsp90-5 & \multirow{2}{*}{ Group Ila } \\
\hline Lsat_1_v5_gn_9_104581.1 & LsHsp90-6 & \\
\hline Lsat_1_v5_gn_8_30781.1 & LsHsp90-7 & Group Illb \\
\hline
\end{tabular}

C

LsHsp90-2b

LsHsp90-2a

-

LsHsp90-7

LsHsp90-5

LsHsp90-6

D

\section{Transcript}

Lsat_1_v5_gn_4_ 110600.1

Lsat_1_v5_gn_4_-116120.1

Lsat_1_v5_-gn_2_87921.1

Lsat_1_v5_gn_3_21960.1

Lsat_1_v5_gn_1_27961.1

Lsat_1_v5_-9n_4_84680.1

Lsat_1_v5_gn_5_15861.1

Lsat_1_v5_-gn_4_85180.1

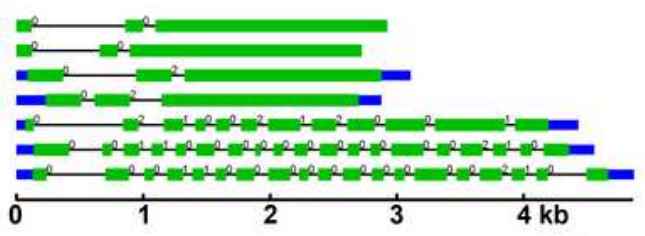

E

$\mathbf{F}$

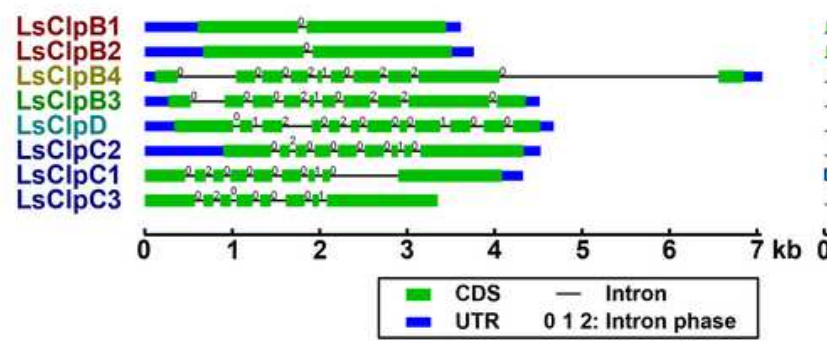

LsClpB1

LsClp82

LsClpB3

LsClpB4
LsClpC1

LsClpC2

LsClpC3

LSCIpD

Subfamily

CplB-Cyt

ClpB-CP

ClpC

ClpD
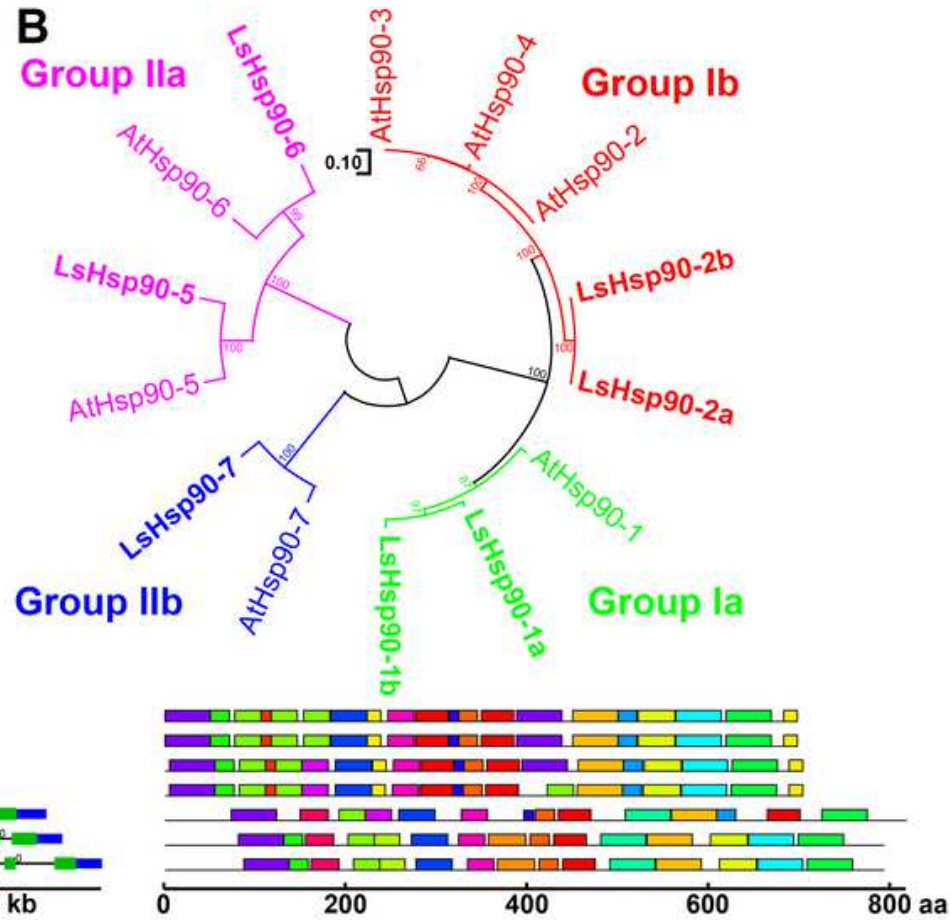

ClpC

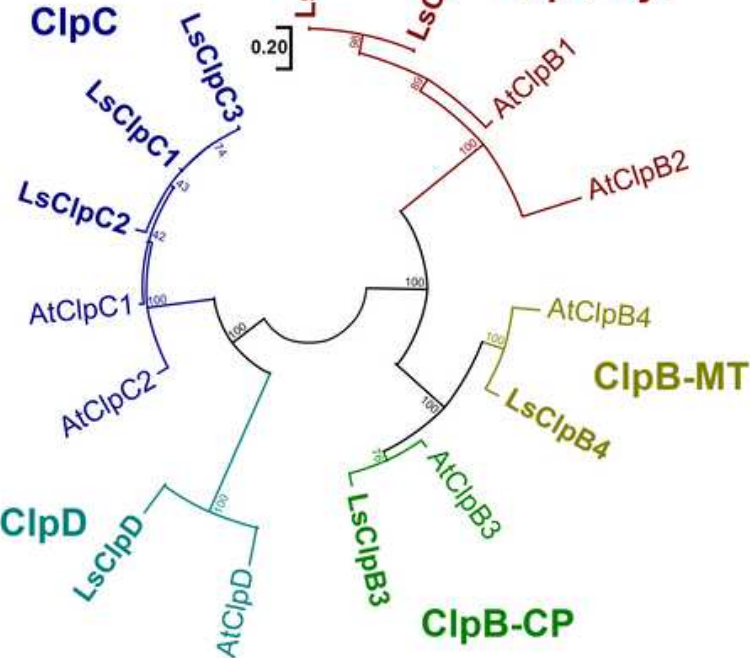

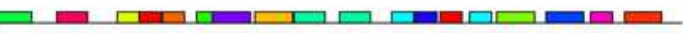

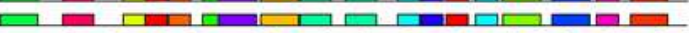

प म

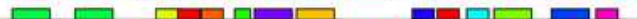
प्रा

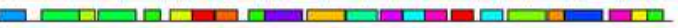

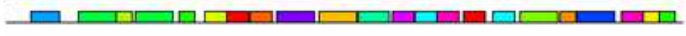

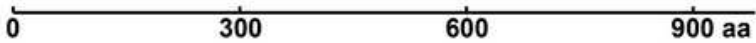

$\square 1 \square 2 \square 3 \quad \square 4 \quad \square 5 \square 6 \quad \square 7 \quad \square 8 \quad \square 9 \square 10$ $\square 11 \square 12 \square 13 \square 14 \square 15 \square 16 \square 17 \square 18 \square 19 \square 20$

Figure 7 
Characterization of lettuce heat shock protein 90 (LsHsp90) and heat shock protein 100 (LsHsp100) gene families. (A) Gene members of the Hsp90 family in lettuce (B). Phylogenetic tree of Hsp90 proteins of L. sativa and A. thaliana. Lettuce Hsp proteins are marked in bold. (C) Gene structure and motif composition analysis of LsHsp90. (D) Gene members of the LsHsp100 family. (E) Phylogenetic analysis of Hsp100 proteins of L. sativa and A. thaliana. (F). Gene structure and motif composition analysis of LsHsp100. Nomenclature of LsHsp90 and LsHsp100 genes were assigned according to the name of A. thaliana homologues. Lettuce Hsp90 and Hsp100 family members are represented in different colors according to their subfamily (Group la, lb, Ila, and Ilb in Hsp90ClpB-Cyt, ClpB-MT, ClpB-CP, ClpC, and ClpD in Hsp100. (C, F) Exon-intron organization. Blue boxes, green boxes, and black lines indicate untranslated region (UTR), coding sequence (CDS), and intron, respectively. The numbers on each intron represent the intron phase $(0,1$, or 2$)$. Detailed information of all motifs is shown in Additional file 3: Table S3. 


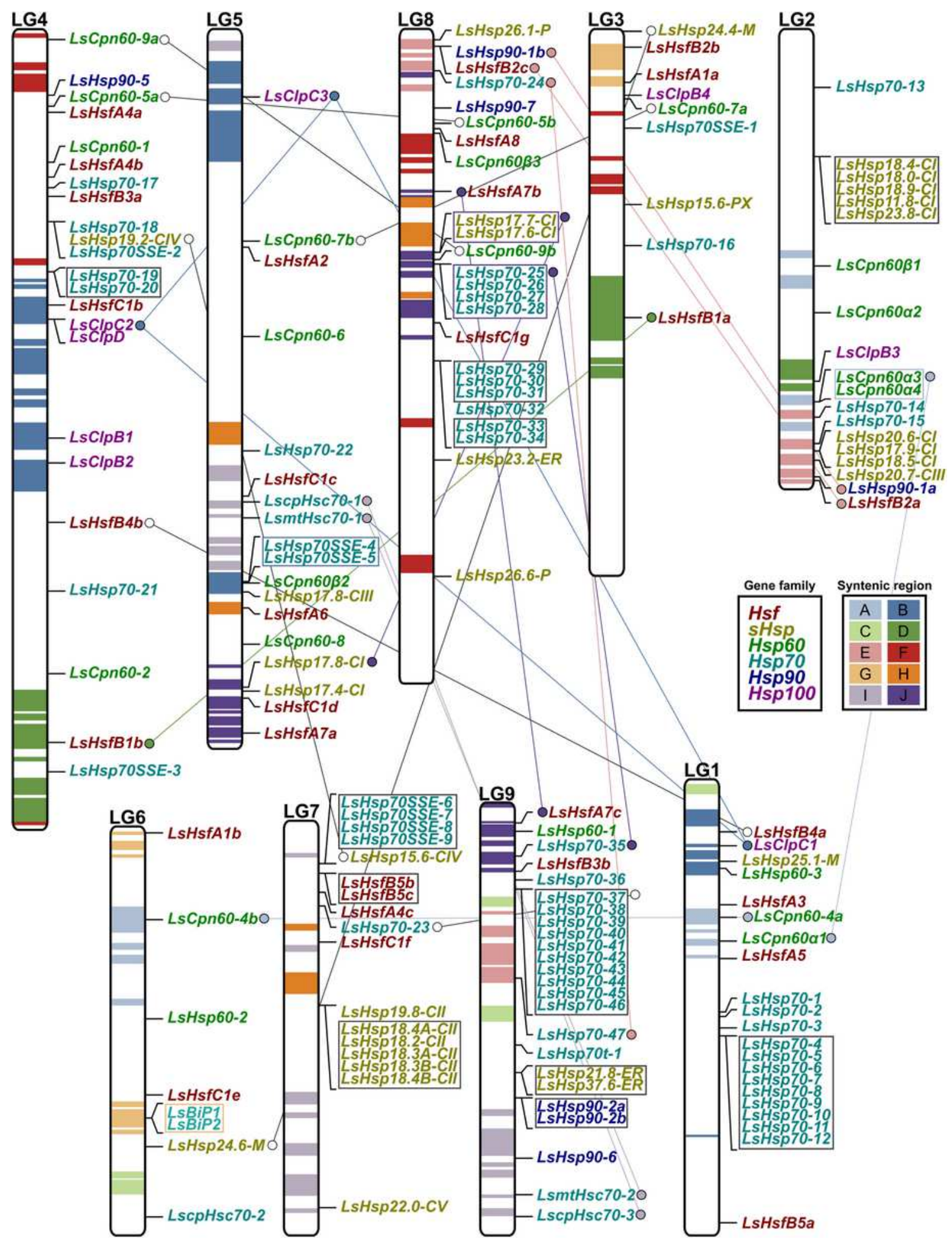

Figure 8

Genome-wide distribution of lettuce triplicated regions highlighting chromosomal location of Hsfs and Hsps genes. Lettuce syntenic regions are shown with colored blocks in each chromosome (LG1 to LG9). Physical location of each LsHsf or LsHsp gene is shown and gene names are colored according to their gene family. LsHsfC1a (Lsat_1_v5_gn_0_11820) was not included because it has not been mapped on 
any of the nine chromosomes in lettuce. Boxes represent tandemly duplicated genes. Paralogous gene pairs originated from segmental duplications are highlighted with circles and connected by lines.

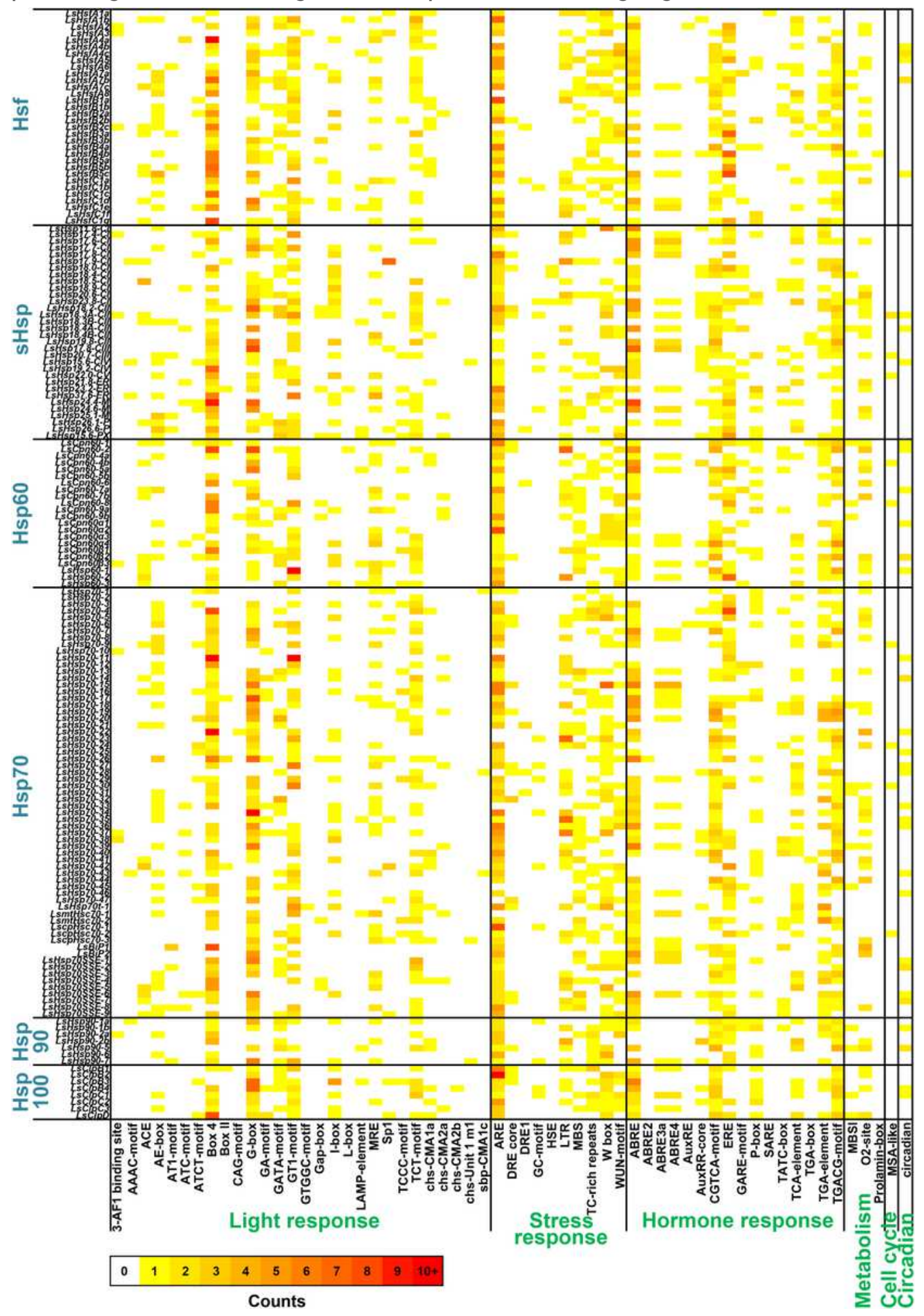

Figure 9

Cis-regulatory element analysis of lettuce Hsf and Hsp genes. Putative cis-regulatory elements were analyzed in the $2 \mathrm{~kb}$ upstream regions of LsHsf and LsHsp genes. Six groups of cis-regulatory elements (light response, stress response, hormone response, metabolism, cell cycle, and circadian rhythm) are 
displayed. Colors from white to red indicate the occurrence of each cis-regulatory element from 0 to $10+$ (10 or more).

A

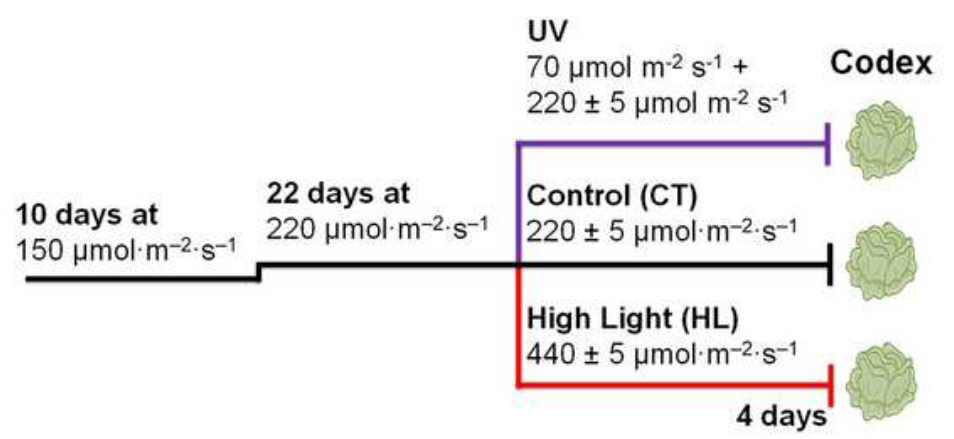

B

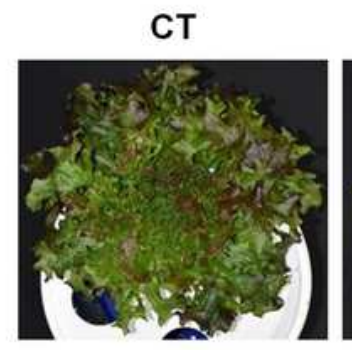

UV

HL

C

UV/CT HL/CT

\begin{tabular}{rl|l} 
& Type A & $\begin{array}{l}\text { LsHsfA1a } \\
\text { LsHsfA7c }\end{array}$ \\
ज & Type B & LsHsfB1a \\
Type C & LsHsfC1C
\end{tabular}

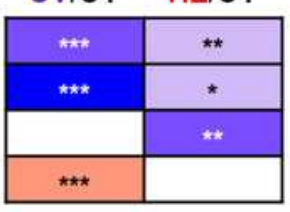

\begin{tabular}{r|l} 
sHsp-CI & $\begin{array}{l}\text { LsHsp17.8-Cl } \\
\text { LsHsp17.9-Cl } \\
\text { LsHsp17.7-Cl }\end{array}$ \\
sHsp-CII & $\begin{array}{l}\text { LsHsp18.2-CII a } \\
\text { LsHsp18.4B-Cll a }\end{array}$
\end{tabular}

은 sHsp-CIII I LsHsp17.8-CIII

sHsp-CV I LsHsp22.0-CV

sHsp-ER I LsHsp23.2-ER

sHsp-MT I LsHsp24.4-M

sHsp-CP I LsHsp26.1-P

sHsp-PX | LsHsp15.6-PX
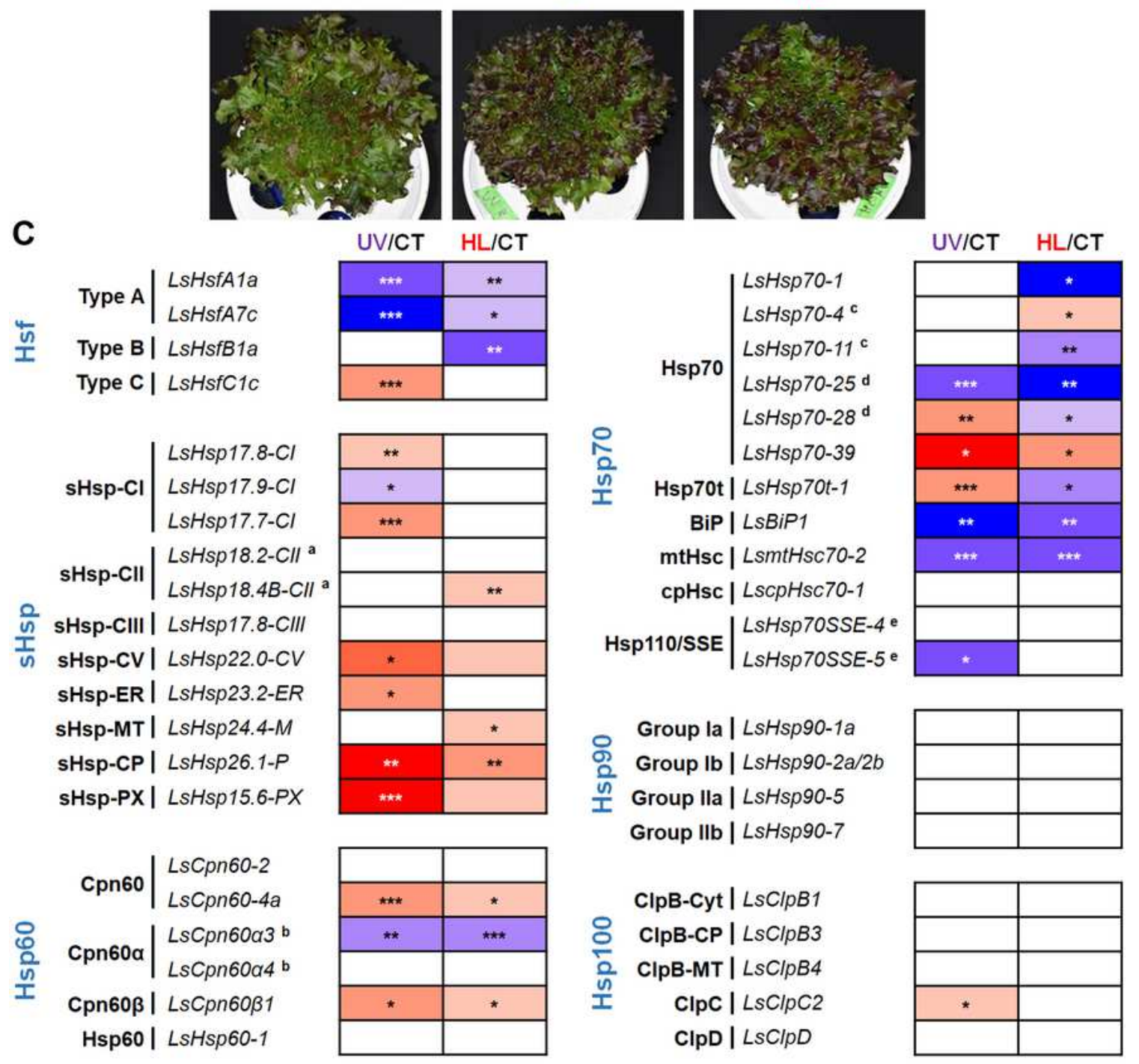

$\left(\log _{2} \mathrm{FC}\right)$

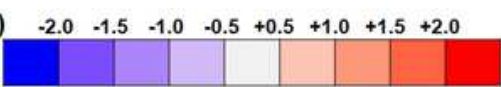

Figure 10

Gene expression analysis of lettuce Hsf and Hsp genes under different light conditions. (A) Schematic representation of the experimental design used at different light treatment on lettuce plants. L. sativa cultivar Codex plants were grown under light $150 \mu \mathrm{mol} \cdot \mathrm{m} \Downarrow 2 \cdot \mathrm{s} \Downarrow 1$ for 10 days then $220 \mu \mathrm{mol} \cdot \mathrm{m} \Downarrow 2 \cdot \mathrm{s} \Downarrow 1$ was 
treated for 22 days. Control light $(\mu \mathrm{mol} \cdot \mathrm{m} \otimes 2 \cdot \mathrm{s} \otimes 1)$, UV light $(11 \mu \mathrm{mol} \cdot \mathrm{m} \otimes 2 \cdot \mathrm{s} \otimes 1$ supplemented with 220 $\mu \mathrm{mol} \cdot \mathrm{m} \otimes 2 \cdot \mathrm{s} \otimes 1$ of white light), and high light $(440 \mu \mathrm{mol} \cdot \mathrm{m} \otimes 2 \cdot \mathrm{s} \otimes 1)$ were imposed to lettuce plants for 4 days. (B) Physical appearance of L. sativa cultivar "Codex" 4 days after treatment of control light (CT), UV, and high light (HL). (C) The relative expression of lettuce Hsf and Hsp genes. mRNA levels were determined by qRT-PCR analysis and normalized to that of LsTUB. log2FC (fold change) values of relative expression of treatment (UV and $\mathrm{HL}$ ) to the control (CT) are presented. Asterisks indicate significant differences between treatment and control determined by Student's t test $(* p<0.05, * \star p<0.01, * \star * p<$ $0.001)$. Genes marked with the same letter (a-e) belong to the same tandem duplicated gene cluster.

\section{Supplementary Files}

This is a list of supplementary files associated with this preprint. Click to download.

- TableS1.ListofHsfandHspgenesandtheircharacteristics.xlsx

- TableS2.dNdSratiobetweenLettucegenesandArabidopsishomologs.xlsx

- TableS3.MEMEMotifanalysis.xlsx

- TableS4.TandemSegmentalduplication.xlsx

- TableS5.CiselementanalysisofLsHsfandLsHsp.xlsx

- TableS6.PrimersusedintheqRTPCRanalysis.xIsx 